\title{
Strong interactive massive particles from a strong coupled theory
}

\author{
Maxim Yu. Khlopov ${ }^{1, *}$ and Chris Kouvaris ${ }^{2, \dagger}$ \\ ${ }^{1}$ Center for Cosmoparticle Physics “Cosmion,” 125047, Moscow, Russia, \\ Moscow Engineering Physics Institute, 115409 Moscow, Russia, \\ and APC Laboratory 10, rue Alice Domon et Léonie Duquet 75205 Paris Cedex 13, France \\ ${ }^{2}$ CERN Theory Division, CH-1211 Geneva 23, Switzerland, \\ University of Southern Denmark, Campusvej 55, DK-5230 Odense, Denmark, \\ and The Niels Bohr Institute, Blegdamsvej 17, DK-2100 Copenhagen, Denmark
}

(Received 15 October 2007; published 3 March 2008)

\begin{abstract}
Minimal walking technicolor models can provide a nontrivial solution for cosmological dark matter, if the lightest technibaryon is doubly charged. Technibaryon asymmetry generated in the early Universe is related to baryon asymmetry, and it is possible to create an excess of techniparticles with charge $(-2)$. These excessive techniparticles are all captured by ${ }^{4} \mathrm{He}$, creating techni-O-helium tOHe atoms, as soon as ${ }^{4} \mathrm{He}$ is formed in big bang nucleosynthesis. The interaction of techni-O-helium with nuclei opens new paths to the creation of heavy nuclei in big bang nucleosynthesis. Because of the large mass of technibaryons, the $t \mathrm{OHe}$ "atomic" gas decouples from the baryonic matter and plays the role of dark matter in large scale structure formation, while structures in small scales are suppressed. Nuclear interactions with matter slow down cosmic techni-O-helium in the Earth below the threshold of underground dark matter detectors, thus escaping severe cryogenic dark matter search constraints. On the other hand, these nuclear interactions are not sufficiently strong to exclude this form of strongly interactive massive particles by constraints from the XQC experiment. Experimental tests of this hypothesis are possible in the search for $t \mathrm{OHe}$ in balloon-borne experiments (or on the ground) and for its charged techniparticle constituents in cosmic rays and accelerators. The $t \mathrm{OHe}$ atoms can cause cold nuclear transformations in matter and might form anomalous isotopes, offering possible ways to exclude (or prove?) their existence.
\end{abstract}

DOI: 10.1103/PhysRevD.77.065002

PACS numbers: $12.60 . \mathrm{Nz}, 95.35 .+\mathrm{d}, 98.80 . \mathrm{Cq}$

\section{INTRODUCTION}

The question of the existence of new quarks and leptons is among the most important in the modern high energy physics. This question has an interesting cosmological aspect. If these quarks and/or charged leptons are stable, they should be present around us and the reason for their evanescent nature should be found.

Recently, at least three elementary particle frames for heavy stable charged quarks and leptons were considered: (a) A heavy quark and heavy neutral lepton (neutrino with mass above half the $Z$-boson mass) of a fourth generation $[1,2]$, which can avoid experimental constraints [3,4], and form composite dark matter species [5-8]; (b) A Glashow's "sinister" heavy tera-quark $U$ and tera-electron $E$, which can form a tower of tera-hadronic and tera-atomic bound states with "tera-helium atoms" (UUUEE) considered as dominant dark matter [9,10]; (c) AC-leptons, based on the approach of almost-commutative geometry [11,12], that can form evanescent $\mathrm{AC}$-atoms, playing the role of dark matter $[11,13,14]$.

In all these recent models, the predicted stable charged particles escape experimental discovery, because they are hidden in elusive atoms, composing the dark matter of the modern Universe. It offers a new solution for the physical

\footnotetext{
*maxim.khlopov@roma1.infn.it

†kouvaris@nbi.dk
}

nature of the cosmological dark matter. Here we show that such a solution is possible in the framework of walking technicolor models [15-20] and can be realized without an ad hoc assumption on charged particle excess, made in the approaches (a)-(c).

This approach differs from the idea of dark matter composed of primordial bound systems of superheavy charged particles and antiparticles, proposed earlier to explain the origin of ultra high energy cosmic rays (UHECR) [21]. To survive to the present time and to be simultaneously the source of UHECR, superheavy particles should satisfy a set of constraints, which, in particular, exclude the possibility that they possess gauge charges of the standard model.

The particles considered here participate in the standard model interactions and we show how the problems, related to various dark matter scenarios with composite atomlike systems, can find an elegant solution on the base of the minimal walking technicolor model.

The approaches (b) and (c) try to escape the problems of free charged dark matter particles [22] by hiding oppositecharged particles in atomlike bound systems, which interact weakly with baryonic matter. However, in the case of charge symmetry, when primordial abundances of particles and antiparticles are equal, annihilation in the early Universe suppresses their concentration. If this primordial abundance still permits these particles and antiparticles to be the dominant dark matter, the explosive nature of such 
dark matter is ruled out by constraints on the products of annihilation in the modern Universe [3,13]. Even in the case of charge asymmetry with primordial particle excess, when there is no annihilation in the modern Universe, binding of positive and negative charged particles is never complete and positively charged heavy species should remain. Recombining with ordinary electrons, these heavy positive species give rise to cosmological abundance of anomalous isotopes, exceeding experimental upper limits. To satisfy these upper limits, the anomalous isotope abundance on Earth should be reduced, and the mechanisms for such a reduction are accompanied by effects of energy release which are strongly constrained, in particular, by the data from large volume detectors.

These problems of composite dark matter models $[9,11]$ revealed in $[3,5,10,13]$, can be avoided, if the excess of only -2 charge $A^{--}$particles is generated in the early Universe. Here we show that in walking technicolor models, technilepton and technibaryon excess is related to baryon excess, and the excess of -2 charged particles can appear naturally for a reasonable choice of model parameters. It distinguishes this case from other composite dark matter models, since in all the previous realizations, starting from [9], such an excess was put by hand to saturate the observed cold dark matter (CDM) density by composite dark matter.

After it is formed in big bang nucleosynthesis, ${ }^{4} \mathrm{He}$ screens the $A^{--}$charged particles in composite $\left({ }^{4} \mathrm{He}^{++} A^{--}\right)$"atoms." These neutral primordial nuclear interacting objects saturate the modern dark matter density and play the role of a nontrivial form of strongly interacting dark matter $[23,24]$. The active influence of this type of dark matter on nuclear transformations seems to be incompatible with the expected dark matter properties. However, it turns out that the considered scenario is not easily ruled out $[5,13]$ and challenges the experimental search for techni-O-helium and its charged techniparticle constituents.

The structure of the present paper is as follows. Starting with a review of possible dark matter candidates offered by the minimal walking technicolor model, we reveal the possibility for the lightest techniparticle(s) to have electric charge \pm 2 (Sec. II). In Sec. III we show how the minimal technicolor model can provide substantial excess of techniparticles with electric charge -2 . In Sec. IV we show how all these -2 charged particles can be captured by ${ }^{4} \mathrm{He}$, after its formation in the standard big bang nucleosynthesis (SBBN), making neutral techni-O-helium atoms that can account for the modern dark matter density. Techni-Ohelium catalyzes a path for heavy element formation in SBBN, but we stipulate in Sec. IV a set of arguments by which the considered scenario can avoid immediate contradiction with observations. A gas of heavy techni-O-helium atoms decouples from the plasma and radiation only at a temperature about few hundreds $\mathrm{eV}$, so that small scale density fluctuations are suppressed and gravitational instability in this gas develops more close to warm dark matter, rather than to the cold dark matter scenario (Sec. VA). We further discuss in Sec. V the possibility to detect charged techniparticle components of cosmic rays (Sec. V B), effects of techni-O-helium catalyzed processes in Earth (Sec. V C), and possibilities of direct searches for techniO-helium (Sec. VD). The problems, signatures, and possible experimental tests of the techni-O-helium Universe are considered in Sec. VI. Details of our calculations are presented in the Appendices A and B.

\section{DARK MATTER FROM WALKING TECHNICOLOR}

The minimal walking technicolor model [15-20] has two techniquarks, i.e., up $U$ and down $D$, that transform under the adjoint representation of an $S U(2)$ technicolor gauge group. The global symmetry of the model is an $S U(4)$ that breaks spontaneously to an $S O(4)$. The chiral condensate of the techniquarks breaks the electroweak symmetry. There are nine Goldstone bosons emerging from the symmetry breaking. Three of them are eaten by the $W$ and the $Z$ bosons. The remaining six Goldstone bosons are $U U, U D, D D$, and their corresponding antiparticles. For completeness $U U$ is $U_{\alpha}^{\top} C U_{\beta} \delta^{\alpha \beta}$, where $C$ is the charge conjugate matrix and the Greek indices denote technicolor states. For simplicity in our notation we omit the contraction of Dirac and technicolor indices. Since the techniquarks are in the adjoint representation of the $S U(2)$, there are three technicolor states. The $U D$ and $D D$ have similar Dirac and technicolor structure. The pions and kaons which are the Goldstone bosons in QCD carry no baryon number since they are made of pairs of quarkantiquark. However in our case, the six Goldstone bosons carry technibaryon number since they are made of two techniquarks or two antitechniquarks. This means that if no processes violate the technibaryon number, the lightest technibaryon will be stable. The electric charges of $U U$, $U D$, and $D D$ are given in general by $y+1, y$, and $y-1$, respectively, where $y$ is an arbitrary real number. For any real value of $y$, gauge anomalies are canceled [20]. The model requires in addition the existence of a fourth family of leptons, i.e., a "new neutrino" $\nu^{\prime}$ and a "new electron" $\zeta$ in order to cancel the Witten global anomaly. Their electric charges are in terms of $y$, respectively $(1-3 y) / 2$ and $(-1-3 y) / 2$. The effective theory of this minimal walking technicolor model has been presented in $[19,25]$.

There are several possibilities for a dark matter candidate emerging from this minimal walking technicolor model. For the case where $y=1$, the $D$ techniquark (and therefore also the $D D$ boson) become electrically neutral. If one assumes that $D D$ is the lightest technibaryon, then it is absolutely stable, because there is no way to violate the technibaryon number apart from the sphalerons that freeze out close to the electroweak scale. This scenario was 
studied in Refs. $[19,20]$. It was shown that $D D$ can provide the full dark matter density if its mass is of the order of $\mathrm{TeV}$. The exact value of the mass of $D D$ depends on the temperature where sphalerons freeze out, and on the ratios $L / B$ and $L^{\prime} / B$, where $L$ and $L^{\prime}$ are the lepton number and the lepton number of the fourth lepton family, respectively, and $B$ is the baryon number. However, this scenario is ruled out by the Cryogenic Dark Matter Search (CDMS) experiment, if $D D$ accounts for $100 \%$ of the dark matter density. The reason is that since $D D$ has a spin independent (SI) interaction with nuclei, it can scatter coherently in underground dark matter detectors, raising the elastic cross section. Such a cross section is already excluded by CDMS, if we accept that the local dark matter density ranges between $0.2-0.4 \mathrm{GeV} / \mathrm{cm}^{3}$. However, if $D D$ is a subdominant component, contributing up to $20 \%$ of the total dark matter density, it cannot yet be ruled out.

Within the same model and electric charge assignment, there is another possibility. Since both techniquarks and technigluons transform under the adjoint representation of the $S U(2)$ group, it is possible to have bound states between a $D$ and a technigluon $G$. The object $D^{\alpha} G^{\alpha}$ (where $\alpha$ denotes technicolor states) is technicolorless. If such an object has a Majorana mass, then it can account for the whole dark matter density without being excluded by CDMS, due to the fact that Majorana particles have no SI interaction with nuclei and their noncoherent elastic cross section is very low for the current sensitivity of detectors [26]. We should emphasize that nonzero Majorana mass means that the technibaryon number is not protected, as in the previous case. For this scenario to be true, the bound state of $D G$ should be lighter than $D D$. The lack of tools in order to study the spectrum of the theory makes it hard to decisively conclude if this is true. Lattice calculations have difficulties to studying objects like $D G$, and evidently perturbation techniques cannot apply. However, in studies of super Yang Mills models with supersymmetry softly broken, it has been argued that a Majorana mass for the gluino makes the $\lambda G$ ( $\lambda$ being the gluino) lighter than the $\lambda \lambda$ [27]. If we transfer directly these results in our case, it would mean that $D G$ is lighter than $D D$ as long as $D$ has a Majorana mass. Of course this argument can be only taken as an indication, since the considered walking technicolor model is not supersymmetric and because $D$ in principle has also a Dirac mass. On the other hand, if the Majorana mass is zero, the above argument cannot be applied. In this case it is more natural to expect that $D D$ (or $U U$ and $U D$ ), which is a Goldstone boson, is the lightest technibaryon. That might imply that $D G$ is unstable.

Finally, if one chooses $y=1 / 3, \nu^{\prime}$ has zero electric charge. In this case the heavy fourth Majorana neutrino $\nu^{\prime}$ can play the role of a dark matter particle. This scenario was explored first in [28] and later in [26]. It was shown that indeed the fourth heavy neutrino can provide the dark matter density without being excluded by CDMS [29] or any other experiment. This scenario allows the possibility for new signatures of weakly interacting massive particle annihilation [30].

In this paper we study a case that resembles mostly the first one mentioned above, that is, $y=1$ and the Goldstone bosons $U U, U D$, and $D D$ have electric charges 2,1 , and 0 , respectively. In addition, for $y=1$, the electric charges of $\nu^{\prime}$ and $\zeta$ are respectively -1 and -2 . We are interested in the case where stable particles with -2 electric charge have substantial relic densities and can capture ${ }^{4} \mathrm{He}^{++}$ nuclei to form a neutral atom. There are three possibilities for this scenario. The first one is to have a relic density of $\bar{U} \bar{U}$, which has -2 charge. For this to be true we should assume that $U U$ is lighter than $U D$ and $D D$ and no processes (apart from electroweak sphalerons) violate the technibaryon number. The second one is to have an abundance of $\zeta$ that again has -2 charge, and the third case is to have both $\bar{U} \bar{U}$ (or $D D$ or $\bar{D} \bar{D}$ ) and $\zeta$. For the first case to be realized, $U U$ although charged, should be lighter than both $U D$ and $D D$. This can happen if one assumes that there is an isospin splitting between $U$ and $D$. This is not hard to imagine since for the same reason in QCD the charged proton is lighter than the neutral neutron. Upon making this assumption, $U D$ and $D D$ will decay through weak interactions to the lightest $U U$. The technibaryon number is conserved and therefore $U U$ (or $\bar{U} \bar{U}$ ) is stable. Similarly in the second case where $\zeta$ is the abundant -2 charge particle, $\zeta$ must be lighter than $\nu^{\prime}$ and there should be no mixing between the fourth family of leptons and the other three of the standard model. The $L^{\prime}$ number is violated only by sphalerons and therefore after the temperature falls roughly below the electroweak scale $\Lambda_{\mathrm{EW}}$ and the sphalerons freeze out, $L^{\prime}$ is conserved, which means that the lightest particle, that is $\zeta$ in this case, is absolutely stable. We assume also that technibaryons decay to standard model particles through extended technicolor (ETC) interactions and therefore $T B=0$. Finally in the third case, we examine the possibility to have both $L^{\prime}$ and $T B$ conserved after sphalerons have frozen out. In this case, the dark matter would be composed of bound atoms $\left({ }^{4} \mathrm{He}^{++} \zeta^{--}\right)$and either $\left({ }^{4} \mathrm{He}^{++}(\bar{U} \bar{U})^{--}\right)$or neutral $D D$ (or $\bar{D} \bar{D}$ ). We shall examine the three possibilities separately in the next section.

\section{THE EXCESS OF THE - 2 CHARGED TECHNIPARTICLES IN THE EARLY UNIVERSE}

The calculation of the excess of the technibaryons with respect to the one of the baryons was pioneered in Refs. [31-33]. In this paper we calculate the excess of $\bar{U} \bar{U}$ and $\zeta$ along the lines of [20]. The technicolor and the standard model particles are in thermal equilibrium as long as the rate of the weak (and color) interactions is larger than the expansion of the Universe. In addition, the sphalerons allow the violation of the technibaryon number $T B$, $B, L$, and $L^{\prime}$ as long as the temperature of the Universe is 
higher than roughly $\Lambda_{\mathrm{EW}}$. It is possible through the equations of thermal equilibrium, sphalerons, and overall electric neutrality for the particles of the Universe, to associate the chemical potentials of the various particles. Following [20], we can write down the $B, T B, L$, and $L^{\prime}$ as

$$
\begin{gathered}
B=12 \mu_{u L}+6 \mu_{W}, \\
T B=\frac{2}{3}\left(\left(\sigma_{U U}+\sigma_{U D}+\sigma_{D D}\right) \mu_{U U}\right. \\
\left.+\left(\sigma_{U D}+2 \sigma_{D D}\right) \mu_{W}\right), \\
L=4 \mu+6 \mu_{W}, \\
L^{\prime}=4 \sigma_{\zeta} \mu_{\nu_{L}^{\prime}}+2 \sigma_{\zeta} \mu_{W},
\end{gathered}
$$

where $\mu_{u L}, \mu_{W}, \mu_{\nu^{\prime}}, \mu_{U U}$ are, respectively, the chemical potentials of the left-handed up quark, $W, \nu^{\prime}$, and $U U . \mu$ is the sum of the chemical potentials of the three left-handed neutrinos and the $\sigma_{\alpha}$ parameters denote statistical factors for the species $\alpha$ defined as

$\sigma_{\alpha}=\frac{6}{4 \pi^{2}} \int_{0}^{\infty} d x x^{2} \cosh ^{-2}\left(\frac{1}{2} \sqrt{x^{2}+\left(\frac{m_{\alpha}}{T^{*}}\right)^{2}}\right)$ for fermions,

$\sigma_{\alpha}=\frac{6}{4 \pi^{2}} \int_{0}^{\infty} d x x^{2} \sinh ^{-2}\left(\frac{1}{2} \sqrt{x^{2}+\left(\frac{m_{\alpha}}{T^{*}}\right)^{2}}\right)$ for bosons,

where $m_{\alpha}$ is the mass of $\alpha$ and $T^{*}$ is the freeze out temperature for the sphaleron. In the derivation we have assumed for simplicity that the mass of $\nu^{\prime}$ and $\zeta$ are very close, so $\sigma_{\nu^{\prime}} \approx \sigma_{\zeta}$, and that the standard model particles are massless at $T>\Lambda_{\mathrm{EW}}$. The sphaleron processes and the condition of the overall electric neutrality impose two extra conditions on the chemical potentials [20],

$$
\begin{gathered}
9 \mu_{u L}+\frac{3}{2} \mu_{U U}+\mu+\mu_{\nu^{\prime}}+8 \mu_{W}=0, \\
Q=6 \mu_{u L}+\left(2 \sigma_{U U}+\sigma_{U D}\right) \mu_{U U}-2 \mu-6 \sigma_{\zeta} \mu_{\nu^{\prime}} \\
+\left(\sigma_{U D}-4 \sigma_{\zeta}-18\right) \mu_{W}+\left(14+\sigma_{\zeta}\right) \mu_{0}=0,
\end{gathered}
$$

where $\mu_{0}$ is the chemical potential of the Higgs boson. Using Eqs. (1)-(4), (7), and (8), we can write the ratio of $T B / B$ as a function of the ratios $L / B, L^{\prime} / B$ and statistical factors as

$$
\frac{T B}{B}=-\sigma_{U U}\left(\frac{L^{\prime}}{B} \frac{1}{3 \sigma_{\zeta}}+1+\frac{L}{3 B}\right)
$$

For the derivation of the above ratio, we have assumed that the electroweak phase transition is of second order, which means that the sphaleron processes freeze out at a temperature slightly lower than the electroweak phase transition. For this reason we have taken $\mu_{0}=0$, since the chemical potential of the Higgs boson in the broken phase should be zero. The calculation in the case of a first order phase transition is slightly different, but the results are very similar to the ones of the second order [20]. Furthermore, we have assumed that the mass differences among $U U$, $U D$, and $D D$ are not large and therefore we have made the approximation $\sigma_{D D} \simeq \sigma_{U D} \simeq \sigma_{U U}$. In principle we do not have to make this approximation. The ratio (9) would look a bit more complicated but it would not change our physical conclusions. The mass differences among $U U, U D$, and $D D$ depend on the isospin splitting of the two techniquarks $U$ and $D$. However, if the splitting is not large, as in the case between up and down quarks in QCD, the mass differences among $U U, U D$, and $D D$ are small compared to the electroweak energy scale and consequently our approximation is justified. We should emphasize two points regarding the ratio (9). The minus sign in the right-hand side denotes the fact that if the quantity inside the parentheses is positive, there is an abundance of antitechnibaryons and not technibaryons. In the first case that we investigate, where $U U$ is lighter than $U D$ and $D D$, an abundance of $\bar{U} \bar{U}$ will provide the charge -2 particles that capture the positively charged nucleus of helium in order to form the neutral dark matter atom. The second point is that (9) seems to diverge if we take the limit where the mass of the $\zeta$ and $\nu^{\prime}$ becomes infinite. In that case $\sigma_{\zeta} \rightarrow 0$ and the ratio diverges. However, $L^{\prime}$ as seen in (4) depends linearly on $\sigma_{\zeta}$ and therefore in the limit where the mass of the $\zeta$ is very large $L^{\prime} \rightarrow 0$, unless $\mu_{\nu^{\prime}} \rightarrow \infty$, which is something unnatural. As we mentioned already there are three different cases that we investigate separately regarding the production of dark matter. The first case is when $\bar{U} \bar{U}$ is the -2 charged particle that will bind with helium to form a neutral atom.

\section{A. The case of $\bar{U} \bar{U}$}

In this case, $\bar{U} \bar{U}$ is the source of -2 charged particles. The ratio of dark matter produced by the neutral bound state of $\left({ }^{4} \mathrm{He}^{++}(\bar{U} \bar{U})^{--}\right)$over the baryon matter is

$$
\frac{\Omega_{\mathrm{DM}}}{\Omega_{B}}=\frac{3}{2} \frac{T B}{B} \frac{m_{o}}{m_{p}},
$$

where $m_{o}$ is the mass of the "dark matter atom," which is approximately the mass of $\bar{U} \bar{U}$ plus $4 \mathrm{GeV}$ (the mass of helium) and $m_{p}$ is the mass of the proton. In Fig. 1 we show the ratio $\Omega_{T B} / \Omega_{B}$ as a function of the mass $m$ of the $\bar{U} \bar{U}$ for several values of the parameter $\xi$ and $T^{*}$ (the sphaleron freeze out temperature). The parameter $\xi$ is defined as

$$
\xi=\frac{L^{\prime}}{3 B \sigma_{\zeta}}+1+\frac{L}{3 B}
$$

We should emphasize here that there are two options regarding the new leptons $\nu^{\prime}$ and $\zeta$. If $\zeta$ is the lightest between the two and below the electroweak scale no 

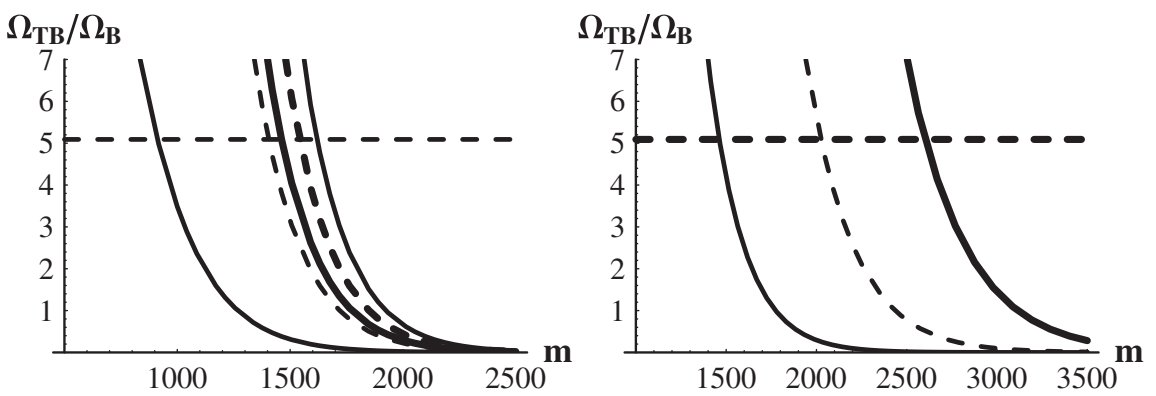

FIG. 1. Left Panel: The ratio of $\Omega_{T B} / \Omega_{B}$ as a function of the mass $m$ (in GeV) of the $\bar{U} \bar{U}$ for several values of $\xi$. The first thin solid line from the left corresponds to $\xi=0.1$, the thin dashed line to $\xi=1$, the thick solid line to $\xi=4 / 3$, the thick dashed line to $\xi=2$, and the last solid line to the right to $\xi=3$. The horizontal dashed line gives the proper ratio of dark matter over baryon matter, which is approximately 5. For all the curves we have set $T^{*}=150 \mathrm{GeV}$. Right Panel: The same ratio for fixed $\xi=4 / 3$, for three different values of the freeze out temperature for the sphalerons $T^{*}$, i.e., $T^{*}=150 \mathrm{GeV}$ (thin solid line), $T^{*}=200 \mathrm{GeV}$ (dashed line), and $T^{*}=250 \mathrm{GeV}$ (thick solid line).

processes violate $L^{\prime}$, then $\zeta$ will contribute to the relic density of -2 charged particles that bind with helium. We study this case later in Sec. III C. If $\nu^{\prime}$ is lighter than $\zeta$, nonzero $L^{\prime}$ could create problems for our model because we have relic density of charged -1 particles. Therefore, we are forced to assume that $L^{\prime}=0$. This is a plausible assumption if one allows mixing between $\nu^{\prime}$ and standard model leptons, because in that case $\nu^{\prime}$ will decay to lighter leptons and $L^{\prime}=0$. Upon making this assumption, i.e., $L^{\prime}=0, \xi=1+L /(3 B)$. In the left panel of Fig. 1 we have chosen $T^{*}=150 \mathrm{GeV}$ and several values of $\xi$ ranging from 0.1 to 3 . In principle $\xi$ can take any positive real value. However, it is logical to assume that the ratio $L / B$ should be around unity. In fact leptogenesis scenarios support a ratio of $L / B=1$. However, since currently it is not possible to know from observations what is the relic density of the light neutrinos (or antineutrinos), $L / B$ can be also negative. As can be seen from the figure, the smaller the value of $\xi$, the lighter becomes the desired $\bar{U} \bar{U}$ that can give the dark matter density. For $\xi=0$, the excess of technibaryons becomes zero within our approximation. We have plotted several values of $\xi$, namely, $\xi=0.1,1$, $4 / 3,2$, and 3. In particular, the value $\xi=4 / 3$ corresponds to $L=B$. We should stress at this point that for our model to work, $\xi$ should be positive. A negative value of $\xi$ would mean that there is an excess for $U U$ (and not $\bar{U} \bar{U}$ ), which is positively charged and being bound with ordinary electrons plays a role of an anomalous helium isotope, severely restricted in experimental searches.

\section{B. The case of $\zeta$}

Equation (9) gives the ratio of $T B / B$ as a function of $L / B$ and $L^{\prime} / B$. It can be trivially inverted as

$$
\frac{L^{\prime}}{B}=-\sigma_{\zeta}\left(\frac{3 T B}{\sigma_{U U} B}+3+\frac{L}{B}\right)
$$

In this subsection we investigate the case where $\zeta$ is the source of -2 charged particles that can be captured by ${ }^{4} \mathrm{He}$. For this to be true, $\zeta$ must be lighter than $\nu^{\prime}$ and after sphalerons have frozen out, no other processes should violate $L^{\prime}$. The term inside the parentheses of (12) should be negative in order to have abundance of $\zeta$ and not anti- $\zeta$. This probably means that a negative ratio $L / B$ is needed. As we mentioned earlier, for our model to be realized, only an abundance of -2 charged or neutral particles is accepted. An abundance of charged particles with different charges would cause a serious problem $[5-8,10,13]$. We study the case where we have an abundance for both technibaryons and $\zeta$ in the next subsection. Here, we look at the case where $T B=0$. This can be realized if below the electroweak scale, ETC processes that violate $T B$ exist. In such a case, the lightest technibaryon will decay to lighter standard model particles and as a result $T B=0$. If $T B=0$, the dark matter density that $\zeta$ can provide is given by

$$
\frac{\Omega_{L^{\prime}}}{\Omega_{B}}=\frac{L^{\prime}}{B} \frac{m_{o}}{m_{p}},
$$

where $m_{o}$ in this case is the mass of $\zeta$ plus the $4 \mathrm{GeV}$ mass of ${ }^{4} \mathrm{He}$. Figure 1 shows also the dark matter that $\zeta$ with mass $m$ can provide, if we take instead of $\xi=1+L /(3 B)$, $\xi=-2-2 L /(3 B)$. For example the curve with $\xi=4 / 3$, that in the previous case corresponded to $L=B$, now corresponds to $L / B=-5$. This identification is possible because although $\sigma_{\zeta}\left(m / T^{*}\right)$ and $\sigma_{U U}\left(m / T^{*}\right)$ are defined through Eqs. (5) and (6), respectively, for $m>500 \mathrm{GeV}$ (with $T^{*}=150 \mathrm{GeV}$ ), the two parameters are approximately equal.

\section{The case of $\zeta$ plus $\bar{U} \bar{U}$ or $D D$ or $\bar{D} \bar{D}$}

The last case we investigate is the one where below the $T^{*}$ temperature no processes violate $T B$ and $L^{\prime}$. This means that the lightest technibaryon and the lightest fourth family lepton are stable objects. In particular, we assume that $\zeta$ is 
lighter than $\nu^{\prime}$. As for the technibaryons there are two options. The first one is to have $D D$ as the lightest technibaryon. In this case, dark matter will be composed of a mixture of neutral $D D$ or $\bar{D} \bar{D}$ and bound $\left({ }^{4} \mathrm{He}^{++} \zeta^{--}\right)$ atoms. The second option is to have $U U$ as the lightest technibaryon. In this case the only acceptable scenario for dark matter is to have a mixture of bound atoms of $\left({ }^{4} \mathrm{He}^{++} \zeta^{--}\right)$and $\left({ }^{4} \mathrm{He}^{++}(\bar{U} \bar{U})^{--}\right)$. Any other combination, for example, an abundance of $U U$ instead of $\bar{U} \bar{U}$ would create problems in our cosmological model since +2 charged $U U$ represents a form of anomalous helium. Equation (12) relates $T B / B$ to $L / B$ and $L^{\prime} / B$. If both the technibaryon and $\zeta$ contribute to dark matter, the ratio of dark matter over baryon matter is given by

$$
\frac{\Omega_{\mathrm{DM}}}{\Omega_{B}}=\frac{\Omega_{T B}}{\Omega_{B}}+\frac{\Omega_{L^{\prime}}}{\Omega_{B}}=5.09 .
$$

The contribution of the technibaryon is

$$
\frac{\Omega_{T B}}{\Omega_{B}}=\frac{3}{2} \frac{|T B|}{B} \frac{m_{T B}}{m_{p}},
$$

where $m_{T B}$ is the mass of the lightest technibaryon (plus $4 \mathrm{GeV}$ ). We have taken the absolute value of $T B$ because if we have abundance of $D D, T B$ is positive, but for $\bar{U} \bar{U}$ or $\bar{D} \bar{D}, T B$ is negative. Similarly by using (12), the contribution of $\zeta$ is

$$
\frac{\Omega_{L^{\prime}}}{\Omega_{B}}=\sigma_{\zeta}\left|\left(3+\frac{L}{B}+\frac{3 T B}{\sigma_{T B} B}\right)\right| \frac{m_{\zeta}}{m_{p}},
$$

where $m_{\zeta}$ is the mass of the $\zeta$ (plus $4 \mathrm{GeV}$ ). The term inside the absolute brackets should be negative, because we want to have an abundance of $\zeta$ and not anti- $\zeta$. Therefore we have to impose the condition

$$
3+\frac{L}{B}+\frac{3 T B}{\sigma_{T B} B}<0 .
$$

Using the above equations we can rewrite Eq. (14) in a more convenient form

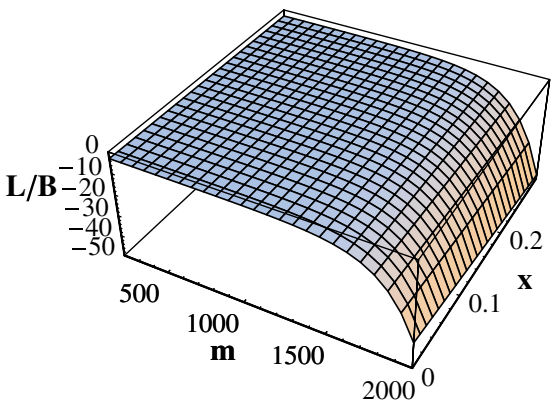

$$
\begin{aligned}
\frac{\Omega_{\mathrm{DM}}}{\Omega_{B}} & =\left|\sigma_{\zeta}\left(3+\frac{L}{B}\right) \frac{m_{\zeta}}{m_{p}} \pm \frac{2 \sigma_{\zeta}}{\sigma_{T B}} \frac{m_{\zeta}}{m_{T B}} 5.09 x\right|+5.09 x \\
& =5.09,
\end{aligned}
$$

where $x$ denotes the fraction of dark matter given by the techibaryon. Again, the term inside the absolute brackets should be negative. If the excessive technibaryon is the $D D$, the above equation should be taken by choosing the plus sign for the term that has \pm . If $\bar{U} \bar{U}$ or $\bar{D} \bar{D}$ is the excessive particle, then the term should be taken with the minus sign.

We first investigate the case of $D D$ mixing. If $D D$ accounts for a component of $x 100 \%$ of the dark matter density, we can express the ratio $L / B$ as a function of $x$ and the masses of $\zeta$ and $D D$. In particular,

$$
\frac{L}{B}=-\frac{5.09 m_{p}}{m_{\zeta} \sigma_{\zeta}}[(2 z-1) x+1]-3,
$$

where $z=\left(\sigma_{\zeta} m_{\zeta}\right) /\left(\sigma_{T B} m_{T B}\right)$. If $m_{\zeta}$ is close to $m_{T B}$, then $z=1$ (provided that the masses are larger than $350 \mathrm{GeV}$, if $\left.T^{*}=150 \mathrm{GeV}\right)$. The study of $D D$ as a dark matter candidate in [20] revealed that $D D$ cannot account for $100 \%$ of dark matter density. If we accept that the local dark matter density in the vicinity of the Earth is between $0.2-0.4 \mathrm{GeV} / \mathrm{cm}^{3}$, then with the current exposure of the detectors in CDMS, $D D$ has been ruled out if it composes $100 \%$ of the dark matter due to its large cross section. However, depending on the mass of $D D$ and the local dark matter density, $D D$ can be a component of dark matter up to 20-30\% without being excluded by CDMS. This means that in Eq. (19), $x$ should be $0<x<0.3$. In Fig. 2, we plot the value of $L / B$ as a function of $m_{\zeta}$ (for $z=1$ ) and $x$ (the fraction of dark matter provided by $D D$ ), in order for the dark matter to be a mixture of $x D D$ and $1-x$ of $\left({ }^{4} \mathrm{He}^{++} \zeta^{--}\right)$. There are three points we would like to stress here. First, it is obvious that the limit $x=0$ corresponds to the case we studied in the previous subsection. Second, as it can be seen from Fig. 2, $L / B$ gets a large negative value very fast as $m_{\zeta}$ increases beyond $1-1.5 \mathrm{TeV}$. This is some-

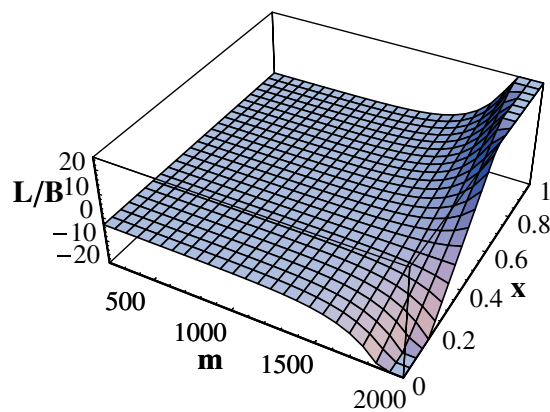

FIG. 2 (color online). Left Panel: The value of $L / B$ in terms of the mass of the $\zeta$ and the fraction of $D D$ in the dark matter density $x$, in order for the composition of dark matter to be $x D D$ and $(1-x)$ of $\left({ }^{4} \mathrm{He}^{++} \zeta^{--}\right)$. Right Panel: Same as in the left panel, for the case where the abundant technibaryon is either $\bar{D} \bar{D}$ or $\bar{U} \bar{U}$. In this case dark matter is made of $(1-x)\left({ }^{4} \mathrm{He}^{++} \zeta^{--}\right)$and $x \bar{D} \bar{D}$ or $\left({ }^{4} \mathrm{He}^{++}(\bar{U} \bar{U})^{--}\right)$. 
thing probably unnatural since it is expected that $L / B$ should be of order unity. Third, we see that as $x$ increases, $L / B$ becomes more negative for constant $m_{\zeta}$. This is because $T B$ is positive and therefore $L / B$ is forced to be more negative in order to get a relic density of $\zeta$. We should also emphasize that if $D D$ is as low as $200 \mathrm{GeV}$, then the CDMS constraint becomes tougher. Taking the strict constraint, for low mass for $D D, x$ should be less than 0.1 or even 0.05 .

We turn now to the case where $T B$ is negative. This means that the excessive technibaryon is either $\bar{D} \bar{D}$ or $\bar{U} \bar{U}$ according to which one is lighter. We can express as before $L / B$ as

$$
\frac{L}{B}=\frac{5.09 m_{p}}{m_{\zeta} \sigma_{\zeta}}[(2 z+1) x-1]-3
$$

In the right panel of Fig. 2 we show again the projected value of $L / B$ in terms of the mass $m_{\zeta}$ and the fraction $x$ of $\bar{D} \bar{D}$ or $\bar{U} \bar{U}$. We plot $x$ from 0 to 1 . The CDMS constraints apply for the $\bar{D} \bar{D}$, but not for $\bar{U} \bar{U}$. This means that if the technibaryon is $\bar{D} \bar{D}, x$ should be at most 0.3 . From the figure we see that in order for $L / B$ to be of order unity, a mass for $\zeta$ and $\bar{U} \bar{U}$ between $1-2 \mathrm{TeV}$ is needed. Again, the limits $x=0$ and $x=1$ were studied in the previous subsections and correspond to the cases of having purely $\left({ }^{4} \mathrm{He}^{++} \zeta^{--}\right)$or purely $\left({ }^{4} \mathrm{He}^{++}(\bar{U} \bar{U})\right)^{--}$, respectively.

The case where both $U U$ and $\zeta$ are stable techniparticles offers another possible dark matter scenario. Positively charged $U U^{++}$and negatively charged $\zeta^{--}$can form atoms $\left(\zeta^{--} U U^{++}\right)$, which behave as cold dark matter species. It resembles the $A^{--} C^{++}$dark matter atoms of the AC-model $[13,14]$. However, as it took place in the ACcosmology, the existence of +2 charged species, which remain free after all the stages of their binding with -2 charged species and ${ }^{4} \mathrm{He}$, has a potential danger of anomalous He overproduction. The solution found in the ACmodel $[13,14]$ for this problem of anomalous He involves an additional strict $U(1)$ gauge symmetry, acting on the AC-leptons, and a new Coulomb-like long range interaction between the AC-leptons, mediated by the corresponding massless $U(1)$ gauge boson. It could be hardly applied to the case of walking technicolor models. One can have another way to solve this problem. The abundance of free +2 charged techniparticles is suppressed, if all of them are bound with -2 charged techniparticles. Such a complete binding takes place naturally, if the excess of -2 charged techniparticles is larger than the one of +2 charged techniparticles. Under these conditions, virtually all +2 charged techniparticles are bound in atoms with -2 charged techniparticles, while the residual -2 charged techniparticles bind with ${ }^{4} \mathrm{He}$ in techni-O-helium. The realization of this scenario in the framework of a walking technicolor model and the nontrivial cosmological scenarios involving both cold $\left(\zeta^{--} U U^{++}\right)$and warm $\left({ }^{4} \mathrm{He} \zeta^{--}\right)$ forms of composite dark matter goes beyond the scope of the present paper, and it is the subject of a separate work.

Because of strong technicolor interactions, the $T B$ excess of $\bar{U} \bar{U}$ suppresses strongly the primordial abundance of the positively charged $U U$ [34]. However in the case of weakly interacting $\zeta$ (in the full analogy with the cases of tera-leptons [10] or AC-leptons [13]), its excess does not guarantee a suppression of the corresponding positively charged antiparticles $\bar{\zeta}$. The stages of cosmological evolution resulting in virtually complete elimination of the primordial $\bar{\zeta}$ are stipulated in Appendix B.

\section{THE CAPTURE OF THE CHARGED TECHNIPARTICLES BY ${ }^{4} \mathrm{He}$}

In the big bang nucleosynthesis, ${ }^{4} \mathrm{He}$ is formed with an abundance $r_{\mathrm{He}}=0.1 r_{\mathrm{B}}=8 \times 10^{-12}$ and, being in excess, binds all the negatively charged technispecies into atomlike systems. Since the electric charge of $\bar{U} \bar{U}$ (and $\zeta$ ) is -2 , neutral atoms are formed, and $\left({ }^{4} \mathrm{He}^{++} \zeta^{--}\right)$atoms catalyze effectively the $(\zeta \bar{\zeta})$ binding and annihilation. It turns out [13] that the electromagnetic cascades from this annihilation cannot influence the light element abundance and the energy release of this annihilation takes place so early that it does not distort the cosmic microwave background (CMB) spectrum.

At a temperature $T<I_{o}=Z_{\mathrm{TC}}^{2} Z_{\mathrm{He}}^{2} \alpha^{2} m_{\mathrm{He}} / 2 \approx$ $1.6 \mathrm{MeV}$, where $\alpha$ is the fine structure constant and $Z_{\mathrm{TC}}=$ -2 stands for the electric charge of $\bar{U} \bar{U}$ and/or of $\zeta$, the reaction

$$
\zeta^{--}+{ }^{4} \mathrm{He}^{++} \rightarrow \gamma+\left({ }^{4} \mathrm{He} \zeta\right)
$$

and/or

$$
(\bar{U} \bar{U})^{--}+{ }^{4} \mathrm{He}^{++} \rightarrow \gamma+\left({ }^{4} \mathrm{He}(\bar{U} \bar{U})\right)
$$

can take place. In these reactions neutral techni-O-helium atoms are produced. The size of these atoms is $[5,13]$

$$
R_{o} \sim 1 /\left(Z_{\mathrm{TC}} Z_{\mathrm{He}} \alpha m_{\mathrm{He}}\right) \approx 2 \times 10^{-13} \mathrm{~cm},
$$

and they can play a nontrivial catalyzing role in nuclear transformations. This aspect needs special thorough study, but some arguments, which we present below following [13], suggest that there should not be contradicting influence on the primordial element abundance.

For our problem another aspect is also important. The reactions (21) and (22) can start only after ${ }^{4} \mathrm{He}$ is formed, which happens at $T<100 \mathrm{keV}$. Then, inverse reactions of ionization by thermal photons support Saha-type relationships between the abundances of these atoms, free -2 charged particles, ${ }^{4} \mathrm{He}$, and $\gamma$ :

$$
\frac{n_{\mathrm{He}} n_{A}}{n_{\gamma} n_{(\mathrm{He} A)}}=\exp \left(-\frac{I_{o}}{T}\right)
$$

From now on, by $A^{--}$we shall denote $\bar{U} \bar{U}$ and $\zeta$, if the 
result is independent of their technibaryon or technilepton nature. When $T$ falls below $T_{r \mathrm{He}} \sim I_{o} / \ln \left(n_{\gamma} / n_{\mathrm{He}}\right) \approx$ $I_{o} / 27 \approx 60 \mathrm{keV}$, free $A^{--}$become bound with helium in the reactions of Eqs. (21) and (22). The fraction of free $A^{--}$, which forms neutral $\left({ }^{4} \mathrm{He}^{++} A^{--}\right)$depends on the ratio of the abundance of $A^{--}$over the one of ${ }^{4} \mathrm{He}$. For $m_{\zeta}>50 \mathrm{GeV}$ and $m_{T B}>50 \mathrm{GeV}$, this ratio is less than 1 . Therefore due to ${ }^{4} \mathrm{He}$ excess, all the $A^{--}$form $\left({ }^{4} \mathrm{He}^{++} A^{--}\right)$atoms through the reactions of Eqs. (21) and (22). Because of this, no free $A^{--}$are left at the time when $T \sim$ few $\mathrm{keV}$, where $\left(p^{+} A^{--}\right)^{-}$"ions" or $\left(p^{+} p^{+} A^{--}\right)$atoms could form.

As soon as techni-O-helium $\left({ }^{4} \mathrm{He}^{++} \zeta^{--}\right)$is formed, heavy antiparticles $\bar{\zeta}$ can penetrate it, expelling ${ }^{4} \mathrm{He}$ and forming $\zeta$-positronium states $(\zeta \bar{\zeta})$, in which the antiparticles annihilate. Therefore the antiparticle $\bar{\zeta}$ can annihilate through formation of positronium, such as

$$
(\mathrm{He} \zeta)+\bar{\zeta} \rightarrow(\zeta \bar{\zeta} \quad \text { annihilation products })+{ }^{4} \mathrm{He} .
$$

\section{A. ${ }^{4} \mathrm{He}$ capture of free negative charges}

At a temperature $T \leq T_{r \mathrm{He}}$, when the reactions of (21) and (22) dominate, the decrease of the free $A^{--}$abundance due to formation of $\left({ }^{4} \mathrm{He}^{++} A^{--}\right)$is governed by the equation [13]

$$
\frac{d r_{A}}{d x}=f_{1 \mathrm{He}}\langle\sigma v\rangle r_{A} r_{\mathrm{He}},
$$

where $x=T / I_{o}, r_{\mathrm{He}}=8 \times 10^{-12},\langle\sigma v\rangle$ is given by

$$
\langle\sigma v\rangle=\left(\frac{4 \pi}{3^{3 / 2}}\right) \cdot \frac{\bar{\alpha}^{2}}{I_{o} \cdot m_{\mathrm{He}}} \frac{1}{x^{1 / 2}},
$$

where $\bar{\alpha}=Z_{\mathrm{TC}} Z_{\mathrm{He}} \alpha$, and (see appendix)

$$
f_{1 \mathrm{He}} \approx m_{P l} I_{o} .
$$

The solution of Eq. (26) is given by [13]

$$
r_{A}=r_{A 0} \exp \left(-r_{\mathrm{He}} J_{\mathrm{He}}\right)=r_{A 0} \exp \left(-1.28 \times 10^{4}\right),
$$

where

$$
\begin{aligned}
J_{\mathrm{He}} & =\int_{0}^{x_{f \mathrm{He}}} f_{1 \mathrm{He}}\langle\sigma v\rangle d x \\
& =m_{P l}\left(\frac{4 \pi}{3^{3 / 2}}\right) \cdot \frac{Z_{\mathrm{TC}}^{2} Z_{\mathrm{He}}^{2} \alpha^{2}}{m_{\mathrm{He}}} \cdot 2 \cdot \sqrt{x_{f \mathrm{He}}} \approx 1.6 \times 10^{15} .
\end{aligned}
$$

$x_{f \mathrm{He}}=1 / 27$ is the value of $T / I_{o}$ where the equilibrium abundance of free charged techniparticles is frozen out and the photodestruction of their atomlike bound states with $\mathrm{He}$ does not prevent recombination, and $m_{P l}$ is the Planck mass. Thus, virtually all the free $A^{--}$are trapped by helium and their remaining abundance becomes exponentially small.

For particles $Q^{-}$with charge -1 , as for tera-electrons in the sinister model [9], ${ }^{4} \mathrm{He}$ trapping results in the formation of a positively charged ion $\left({ }^{4} \mathrm{He}^{++} Q^{-}\right)^{+}$, result in dramatic overproduction of anomalous hydrogen [10]. Therefore, only the choice of -2 electric charge for stable techniparticles makes it possible to avoid this problem. In this case, ${ }^{4} \mathrm{He}$ trapping leads to the formation of neutral OLe-helium atoms $\left({ }^{4} \mathrm{He}^{++} A^{--}\right)$, which can catalyze the complete elimination of primordial positively charged antitechnileptons.

\section{B. Complete elimination of antiparticles by techni-O-helium catalysis}

For large $m_{\zeta},{ }^{1}$ the primordial abundance of antiparticles $\bar{\zeta}$ is not suppressed. The presence of techni-O-helium $\left({ }^{4} \mathrm{He}^{++} \zeta^{--}\right)$in this case accelerates the annihilation of these antiparticles through the formation of $\zeta$-positronium. Similar to the case of tera-particles considered in [10] and AC-leptons considered in [13], it can be shown that the products of annihilation cannot cause a backreaction, ionizing techni-O-helium and suppressing the catalysis.

Indeed, energetic particles, created in $(\zeta \bar{\zeta})$ annihilation, interact with the cosmological plasma. In the development of the electromagnetic cascade, the creation of electronpositron pairs in the reaction $\gamma+\gamma \rightarrow e^{+}+e^{-}$plays an important role in astrophysical conditions (see [35-37] for a review). The threshold of this reaction puts an upper limit on the energy of the nonequilibrium photon spectrum in the cascade

$$
E_{\max }=a \frac{m_{e}^{2}}{25 T}
$$

where the factor $a=\ln \left(15 \Omega_{B}+1\right) \approx 0.5$ [13].

At a temperature $T>T_{r b \mathrm{He}}=a m_{e}^{2} /\left(25 I_{o}\right) \approx 1 \mathrm{keV}$, in the spectrum of the electromagnetic cascade from $(\zeta \bar{\zeta})$ annihilation, the maximal energy $E_{\max }<I_{o}$ and the annihilation products cannot ionize $\left({ }^{4} \mathrm{He}^{++} A^{--}\right)$. So, there is no backreaction of the $(\zeta \bar{\zeta})$ annihilation until $T \sim T_{r b \mathrm{He}}$. At that time, practically all free $\zeta$ and $\bar{U} \bar{U}$ are bound into $\left({ }^{4} \mathrm{He}^{++} A^{--}\right)$atoms. For the same reason, electromagnetic showers induced by annihilation products, having a maximal energy below the binding energies of the SBBN nuclei, cannot initiate reactions of nonequilibrium nucleosynthesis and influence the abundance of light elements.

In the absence of backreaction of annihilation products, nothing prevents the complete elimination of antiparticles $\bar{\zeta}^{++}$by techni-O-helium catalysis. The $\bar{\zeta}^{++}$with primordial abundance $r_{\bar{\zeta}}$, can be captured by techni-O-helium $\left({ }^{4} \mathrm{He}^{++} \zeta^{--}\right)$with abundance $r_{\zeta \mathrm{He}}=r_{\zeta}=r_{\bar{\zeta}}+\kappa$ (here the technilepton excess $\kappa=4 \times 10^{-12} f_{\zeta} / S_{2}$ is given by Eq. (B2) of Appendix B with $0 \leq f_{\zeta} \leq 1$ being the relative contribution of technileptons into the total dark matter density, and $S_{2}$ is the mass of $\zeta$ in units of $100 \mathrm{GeV}$ ). By definition, $f_{\zeta}=1-x$, where $x$ was defined in Eq. (18).

\footnotetext{
${ }^{1}$ From now on $m_{\zeta}$ represents the mass of $\zeta$ and not the mass of $\zeta$ plus $4 \mathrm{GeV}$.
} 
The $\bar{\zeta}^{++}$expels the ${ }^{4} \mathrm{He}$ from the $\left({ }^{4} \mathrm{He}^{++} \zeta^{--}\right)$and annihilates in $\zeta$-positronium $\left(\bar{\zeta}^{++} \zeta^{--}\right)$.

The process of $\bar{\zeta}^{++}$capture by the $\left({ }^{4} \mathrm{He}^{++} \zeta^{--}\right)$atom looks as follows [13]. Being in thermal equilibrium with the plasma, the free $\bar{\zeta}^{++}$have momentum $k=\sqrt{2 T m_{\zeta}}$. If their wavelength is much smaller than the size of the $\left(\mathrm{He}^{++} \zeta^{--}\right)$atom, they can penetrate inside the atom and bind with $A^{--}$, expelling the ${ }^{4} \mathrm{He}$ from it. The rate of this process is determined by the size of the $\left(\mathrm{He}^{++} \zeta^{--}\right)$atoms and is given by

$$
\langle\sigma v\rangle_{0} \sim \pi R_{o}^{2} \sim \frac{\pi}{\left(\bar{\alpha} m_{\mathrm{He}}\right)^{2}}=\frac{\pi}{2 I_{o} m_{\mathrm{He}}} \approx 3 \times 10^{-15} \frac{\mathrm{cm}^{3}}{\mathrm{~s}} .
$$

Here $\quad \bar{\alpha}=Z_{\zeta} Z_{\mathrm{He}} \alpha$. At temperature $T<T_{a}=$ $\bar{\alpha}^{2} m_{\mathrm{He}} \frac{m_{\mathrm{He}}}{2 m_{\zeta}}=\frac{I_{o} m_{\mathrm{He}}}{m_{\zeta}}=4 \times 10^{-2} I_{o} / S_{2}$, the wavelength $\lambda=$ $1 / k$ of $\bar{\zeta}^{++}$exceeds the size $R_{o}=1 /\left(\bar{\alpha} m_{\zeta}\right)$ of the $\left(\mathrm{He}^{++} \zeta^{--}\right)$"atom." The rate of the $\left(\mathrm{He}^{++} \zeta^{--}\right)$catalysis is suppressed by a factor $\left(R_{o} / \lambda\right)^{3}=\left(T / T_{a}\right)^{3 / 2}$ and is given by

$$
\langle\sigma v\rangle_{\text {cat }}\left(T<T_{a}\right)=\langle\sigma v\rangle_{0}\left(T / T_{a}\right)^{3 / 2} .
$$

The decrease of the antiparticle abundance $r_{\bar{\zeta}}$ is described by

$$
\frac{d r_{\bar{\zeta}}}{d x}=f_{1 \mathrm{He}}\langle\sigma v\rangle r_{\bar{\zeta}}\left(r_{\bar{\zeta}}+\kappa\right)
$$

where $x=T / I_{o}, r_{\mathrm{He} \zeta}=r_{\zeta},\langle\sigma v\rangle$ is given by Eq. (30) at $T>T_{a}$ and Eq. (31) at $T<T_{a}$. The solution of this equation is given in [13] and has the form

$$
r_{\bar{\zeta}}=\frac{\kappa \cdot r_{f \bar{\zeta}}}{\left(\kappa+r_{f \bar{\zeta}}\right) \exp \left(\kappa J_{o}\right)-r_{f \bar{\zeta}}},
$$

where $r_{f \bar{\zeta}}$ is the frozen concentration of $\bar{\zeta}$ and

$$
\begin{aligned}
J_{o} & =\int_{0}^{x_{f \mathrm{He}}} f_{1 \mathrm{He}}\langle\sigma v\rangle d x=m_{P l}\left(\frac{\pi}{2 m_{\mathrm{He}}}\right) \cdot x_{f \mathrm{He}} \\
& \approx 1.4 \times 10^{17},
\end{aligned}
$$

where $x_{f \mathrm{He}}=1 / 27$. The factor in the exponent is $\kappa J_{o}=$ $6 \times 10^{5} f_{\zeta} / S_{2}$. It leads to a huge exponential suppression of the antiparticles at all reasonable values of $\kappa$ and $S_{2}$.

\section{Techni-O-helium in the SBBN}

The formation of techni-O-helium reserves a fraction of ${ }^{4} \mathrm{He}$ and thus it changes the primordial abundance of ${ }^{4} \mathrm{He}$. For the lightest possible masses of the techniparticles $m_{\zeta} \sim$ $m_{T B} \sim 100 \mathrm{GeV}$, this effect can reach $50 \%$ of the ${ }^{4} \mathrm{He}$ abundance formed in SBBN. Even if the mass of the techniparticles is of the order of $\mathrm{TeV}, 5 \%$ of the ${ }^{4} \mathrm{He}$ abundance is hidden in the techni-O-helium atoms. This can lead to important consequences once we compare the SBBN theoretical predictions to observations.
The question of the participation of techni-O-helium in nuclear transformations and its direct influence on the chemical element production is less evident. Indeed, techni-O-helium looks like an $\alpha$ particle with a shielded electric charge. It can closely approach nuclei due to the absence of a Coulomb barrier. Because of this, it seems that in the presence of techni-O-helium, the character of SBBN processes should change drastically. However, it might not be the case.

The following simple argument can be used to indicate that the techni-O-helium influence on SBBN transformations might not lead to binding of $A^{--}$with nuclei heavier than ${ }^{4} \mathrm{He}$. In fact, the size of techni-O-helium is of the order of the size of ${ }^{4} \mathrm{He}$ and for a nucleus ${ }_{Z}^{A} Q$ with electric charge $Z>2$, the size of the Bohr orbit for an $Q A^{--}$ion is less than the size of the nucleus ${ }_{Z}^{A} Q$. This means that while binding with a heavy nucleus, $A^{--}$penetrates it and interacts effectively with a part of the nucleus of a size less than the corresponding Bohr orbit. This size corresponds to the size of ${ }^{4} \mathrm{He}$, making techni-O-helium the most bound $Q A^{--}$atomic state. It favors a picture, according to which a techni-O-helium collision with a nucleus, results in the formation of techni-O-helium and the whole process looks like an elastic collision.

The interaction of the ${ }^{4} \mathrm{He}$ component of $\left(\mathrm{He}^{++} A^{--}\right)$ with a ${ }_{Z}^{A} Q$ nucleus can lead to a nuclear transformation due to the reaction

$$
{ }_{Z}^{A} Q+(\mathrm{He} A) \rightarrow{ }_{Z+2}^{A+4} Q+A^{--},
$$

provided that the masses of the initial and final nuclei satisfy the energy condition

$$
M(A, Z)+M(4,2)-I_{o}>M(A+4, Z+2)
$$

where $I_{o}=1.6 \mathrm{MeV}$ is the binding energy of techni-Ohelium and $M(4,2)$ is the mass of the ${ }^{4} \mathrm{He}$ nucleus.

This condition is not valid for stable nuclei participating in reactions of the SBBN. However, tritium, ${ }^{3} \mathrm{H}$, which is also formed in SBBN with abundance ${ }^{3} \mathrm{H} / \mathrm{H} \sim 10^{-7}$ satisfies this condition and can react with techni-O-helium, forming ${ }^{7} \mathrm{Li}$ and opening the path of successive techni-Ohelium catalyzed transformations to heavy nuclei. This effect might strongly influence the chemical evolution of matter on the pregalactic stage and needs a self-consistent consideration within the big bang nucleosynthesis network. However, the following arguments show that this effect may not lead to immediate contradiction with observations as it might be expected.

(i) On the path of reactions (35), the final nucleus can be formed in the excited $(\alpha, M(A, Z))$ state, which can rapidly experience an $\alpha$-decay, giving rise to techni$O$-helium regeneration and to an effective quasielastic process of $\left({ }^{4} \mathrm{He}^{++} A^{--}\right)$-nucleus scattering. It leads to a possible suppression of the techni-Ohelium catalysis of nuclear transformations. 
(ii) The path of reactions (35) does not stop on ${ }^{7} \mathrm{Li}$ but goes further through ${ }^{11} \mathrm{~B},{ }^{15} \mathrm{~N},{ }^{19} \mathrm{~F}, \ldots$ along the table of the chemical elements.

(iii) The cross section of reactions (35) grows with the mass of the nucleus, making the formation of the heavier elements more probable and moving the main output away from a potentially dangerous $\mathrm{Li}$ and $\mathrm{B}$ overproduction.

The first publications on possible realistic composite dark matter scenarios $[5,13]$ gave rise to the development of another aspect of the problem, the charged massive particles BBN (CBBN), studying the influence of unstable negatively charged massive particles in BBN [38-46]. The important difference of CBBN considered in these papers, from our approach, is that singly charged particles $X^{-}$with charge -1 do not screen the +2 charge of $\mathrm{He}$ in a $(\mathrm{HeX})^{+}$ ionlike bound system, and the Coulomb barrier of the $(\mathrm{HeX})^{+}$ion can strongly hamper the path for the creation of isotopes, heavier than ${ }^{6} \mathrm{Li}$. Therefore, ${ }^{6} \mathrm{Li}$ created in the $D+(\mathrm{He} X)$ reaction cannot dominantly transform into heavier elements and if not destructed by $X$-decay products, it should remain in the primordial chemical content. It makes the ${ }^{6} \mathrm{Li}$ overproduction found in [38] a really serious trouble for a wide range of parameters for unstable $X$ particles.

It should be noted that the approach of [38] is not supported by [40]. Moreover, we can mention the following effects [13], missed in its solution for the ${ }^{7} \mathrm{Li}$ problem: (i) the competitive process of ${ }^{7} \mathrm{Li}$ creation by a similar mechanism in the reaction ${ }^{3} \mathrm{H}+(\mathrm{HeX})^{+}$with tritium and (ii) the effects of nonequilibrium nucleosynthesis reactions, induced by hadronic and electromagnetic cascades from products of $X$ decays. The latter effect, which was discussed in [40], implies a self-consistent treatment based on the theory of nonequilibrium cosmological nucleosynthesis $[37,47,48]$ (see also [49-52]). Both effects (i) and (ii) were not studied in [38].

The amount of techni-O-helium in our scenario formally exceeds by a few orders of magnitude the constraint $n_{X} / s \leq 10^{-17}$, derived for concentration $n_{X}$ of metastable $X^{-}$particles in the units of entropy density $s$ in Eq. (10) of [38]. However, it should be noted that this constraint is not valid for our case if the binding energy $I_{o}=1589 \mathrm{keV}$ of techni-O-helium is taken into account. According to [40], this approximation is valid for $0<Z Z_{\mathrm{TC}} \alpha M_{Z} R_{Z}<1$, where $R_{Z} \sim 1.2 A^{1 / 3} / 200 \mathrm{MeV}^{-1}$ is the size of nucleus, which is the case for the $(\mathrm{He} A)$ atom. Then the $D+$ $(\mathrm{He} A)->^{6} \mathrm{Li}+A$ reaction, which the constraint is based on, does not occur. This reaction can take place only if the account for charge distribution in the He nucleus [38] reduces the binding energy of $(\mathrm{He} A)$ down to $E=$ $1200 \mathrm{keV}$ or $E=1150 \mathrm{keV}$ as discussed in [13]. Then this channel becomes possible, but similar to the case of tritium, the chain of techni-O-helium transformations (35), started from deuterium does not stop on ${ }^{6} \mathrm{Li}$, but goes further through ${ }^{10} \mathrm{~B},{ }^{14} \mathrm{~N},{ }^{18} \mathrm{~F}, \ldots$ along the table of the chemical elements. Such a qualitative change of the physical picture appeals to necessity in a detailed nuclear physics treatment of the $\left(A^{--}+\right.$nucleus $)$systems and of the whole set of transformations induced by techni-O-helium, including an analysis of possible fast conversion of helium to carbon and of the formation of a $\left({ }^{8} \mathrm{Be} A^{--}\right)$system, discussed in [13] as potential dangers for our approach. However, one can offer a simple argument, indicating that unlike the CBBN case, there is no problem with ${ }^{6} \mathrm{Li}$ overproduction in nuclear transformations, catalyzed by neutral techni-O-helium. Indeed, this neutral catalyzer can be captured by ${ }^{6} \mathrm{Li}$ with the rate of the order of Eq. (30), while the decrease of its abundance due to the reaction with techni-O-helium is given by an equation of the form of Eq. (26), with the solution similar to Eq. (27),

$$
r_{\mathrm{Li}}=r_{\mathrm{Li} 0} \exp \left(-r_{o} J_{o}\right)=r_{\mathrm{Li} 0} \exp \left(-5.6 \times 10^{5} / S_{2}\right),
$$

where $r_{o}=4 \times 10^{12} / S_{2}$ is the abundance of techni-Ohelium and the integral factor $J_{o}=1.4 \times 10^{17}$ is given by Eq. (34). A similar result can be obtained for ${ }^{10} \mathrm{~B}$, produced in the reaction of ${ }^{6} \mathrm{Li}$ with techni-O-helium, for ${ }^{14} \mathrm{~N}$, produced in the reaction of ${ }^{10} \mathrm{~B}$ with techni-O-helium, etc., until unstable heavy elements appear on the path of these transformations and the backreaction of nuclear decays should be taken into account. Though the above arguments seem to make our approach safe from immediate contradictions with observations, a detailed study of this complicated problem is needed.

\section{TECHNI-O-HELIUM UNIVERSE}

\section{A. Gravitational instability of the techni-O-helium gas}

Because of nuclear interactions of its helium constituent with nuclei in cosmic plasma, the techni-O-helium gas is in thermal equilibrium with plasma and radiation on the radiation dominance (RD) stage, and the energy and momentum transfer from the plasma is effective. The radiation pressure acting on plasma is then effectively transferred to density fluctuations of techni-O-helium gas and transforms them in acoustic waves at scales up to the size of the horizon. However, as it was first noticed in [5], this transfer to heavy nuclear-interacting species becomes ineffective before the end of the RD stage and such species decouple from plasma and radiation. Consequently, nothing prevents the development of gravitational instability in the gas of these species. This argument is completely applicable to the case of techni-O-helium.

At temperature $T<T_{o d} \approx 45 S_{2}^{2 / 3} \mathrm{eV}$, first estimated in [5] for the case of O-helium, the energy and momentum transfer from baryons to techni-O-helium is not effective because $n_{B}\langle\sigma v\rangle\left(m_{p} / m_{o}\right) t<1$, where $m_{o}$ is the mass of the $t \mathrm{OHe}$ atom and $S_{2}=\frac{m_{o}}{100 \mathrm{GeV}}$. Here

$$
\sigma \approx \sigma_{o} \sim \pi R_{o}^{2} \approx 10^{-25} \mathrm{~cm}^{2},
$$


and $v=\sqrt{2 T / m_{p}}$ is the baryon thermal velocity. The techni-O-helium gas decouples from the plasma and plays the role of dark matter, which starts to dominate in the Universe at $T_{\mathrm{RM}}=1 \mathrm{eV}$.

Because of the excess of the ${ }^{4} \mathrm{He}$ abundance over the abundance of techniparticles, the amount of free techniparticles which are not captured by helium is exponentially small (see Sec. IVA). Neutral techni-O-helium atoms cannot have Coulomb interactions with charged particles of matter plasma (electrons and nuclei). The electric dipole moment of the O-helium atom (that is in an $s$-wave ground state) is zero, while the induced dipole moment is strongly suppressed at $T_{o d}<1 \mathrm{keV}$. The only unshielded source of O-helium electromagnetic interaction can be the anomalous magnetic moment of $\zeta$, which is suppressed by the large mass of this particle. Therefore the electromagnetic interaction with plasma is not important in momentum and energy transfer to the techni-O-helium gas.

The development of gravitational instabilities of the techni-O-helium gas triggers large scale structure formation, and the composite nature of techni-O-helium makes it more close to warm dark matter.

The total mass of the $t \mathrm{OHe}$ gas with density $\rho_{d}=$ $\frac{T_{\mathrm{RM}}}{T_{o d}} \rho_{\mathrm{tot}}$ within the cosmological horizon $l_{h}=t$ is

$$
M=\frac{4 \pi}{3} \rho_{d} t^{3}
$$

In the period of decoupling $T=T_{o d}$, this mass depends strongly on the techniparticle mass $S_{2}$ and is given by

$$
\begin{aligned}
M_{o d} & =\frac{T_{\mathrm{RM}}}{T_{o d}} m_{P l}\left(\frac{m_{P l}}{T_{o d}}\right)^{2} \approx 2 \times 10^{46} S_{2}^{-8 / 3} \mathrm{~g} \\
& =10^{13} S_{2}^{-8 / 3} M_{\odot},
\end{aligned}
$$

where $M_{\odot}$ is the solar mass. The techni-O-helium is formed only at $T_{r \mathrm{He}}$ and its total mass within the cosmological horizon in the period of its creation is $M_{o}=$ $M_{o d}\left(T_{o} / T_{o d}\right)^{3}=10^{37} \mathrm{~g}$.

On the RD stage before decoupling, the Jeans length $\lambda_{J}$ of the $t \mathrm{OHe}$ gas was of the order of the cosmological horizon $\lambda_{J} \sim l_{h} \sim t$. After decoupling at $T=T_{o d}$, it falls down to $\lambda_{J} \sim v_{o} t$, where $v_{o}=\sqrt{2 T_{o d} / m_{o}}$. Though after decoupling the Jeans mass in the $t \mathrm{OHe}$ gas correspondingly falls down

$$
M_{J} \sim v_{o}^{3} M_{o d} \sim 3 \times 10^{-14} M_{o d},
$$

one should expect strong suppression of fluctuations on scales $M<M_{o}$, as well as adiabatic damping of sound waves in the RD plasma for scales $M_{o}<M<M_{o d}$. It provides suppression of small scale structure in the considered model for all reasonable masses of techniparticles.

The cross section of mutual collisions of techni-Ohelium atoms is given by Eq. (37). The $t \mathrm{OHe}$ atoms can be considered as collisionless gas in clouds with a number density $n_{o}$ and a size $R$, if $n_{o} R<1 / \sigma_{o}$. This condition is valid for the techni-O-helium gas in galaxies.

Mutual collisions of techni-O-helium atoms determine the evolution timescale for a gravitationally bound system of collisionless $t \mathrm{OHe}$ gas

$$
t_{e v}=1 /\left(n \sigma_{o} v\right) \approx 2 \times 10^{20}\left(1 \mathrm{~cm}^{-3} / n\right)^{7 / 6} \mathrm{~s},
$$

where the relative velocity $v=\sqrt{G M / R}$ is taken for a cloud of mass $M_{o}$ and an internal number density $n$. This timescale exceeds substantially the age of the Universe and the internal evolution of techni-O-helium clouds cannot lead to the formation of dense objects. Being decoupled from baryonic matter, the $t \mathrm{OHe}$ gas does not follow the formation of baryonic astrophysical objects (stars, planets, molecular clouds ...) and forms dark matter halos of galaxies.

\section{B. Techniparticle component of cosmic rays}

The nuclear interaction of techni-O-helium with cosmic rays gives rise to ionization of this bound state in the interstellar gas and to acceleration of free techniparticles in the Galaxy. During the lifetime of the Galaxy $t_{G} \approx 3 \times$ $10^{17} \mathrm{~s}$, the integral flux of cosmic rays

$$
F\left(E>E_{0}\right) \approx 1 \cdot\left(\frac{E_{0}}{1 \mathrm{GeV}}\right)^{-1.7} \mathrm{~cm}^{-2} \mathrm{~s}^{-1}
$$

can disrupt the fraction of galactic techni-O-helium $\sim F\left(E>E_{\min }\right) \sigma_{o} t_{G} \leq 10^{-3}$, where we took $E_{\min } \sim I_{o}$. Assuming a universal mechanism of cosmic ray acceleration, a universal form of their spectrum, taking into account that the ${ }^{4} \mathrm{He}$ component corresponds to $\sim 5 \%$ of the proton spectrum, and that the spectrum is usually reduced to the energy per nucleon, the anomalous low $Z / A-2$ charged techniparticle component can be present in cosmic rays at a level of

$$
\frac{A^{--}}{\mathrm{He}} \geq 3 \times 10^{-7} \cdot S_{2}^{-3.7}
$$

This flux may be within the reach for PAMELA and AMS02 cosmic ray experiments.

Recombination of free techniparticles with protons and nuclei in the interstellar space can give rise to radiation in the range from few tens of $\mathrm{keV}-1 \mathrm{MeV}$. However such a radiation is below the cosmic nonthermal electromagnetic background radiation observed in this range.

\section{Effects of techni-O-helium catalyzed processes in the Earth}

The first evident consequence of the proposed excess is the inevitable presence of $t \mathrm{OHe}$ in terrestrial matter. This is because terrestrial matter appears opaque to $t \mathrm{OHe}$ and stores all its in-falling flux. 
If the $t \mathrm{OHe}$ capture by nuclei is not effective, its diffusion in matter is determined by elastic collisions, which have a transport cross section per nucleon

$$
\sigma_{\mathrm{tr}}=\pi R_{o}^{2} \frac{m_{p}}{m_{o}} \approx 10^{-27} / S_{2} \mathrm{~cm}^{2} .
$$

In atmosphere, with effective height $L_{\text {atm }}=10^{6} \mathrm{~cm}$ and baryon number density $n_{B}=6 \times 10^{20} \mathrm{~cm}^{-3}$, the opacity condition $n_{B} \sigma_{\text {tr }} L_{\text {atm }}=6 \times 10^{-1} / S_{2}$ is not strong enough. Therefore, the in-falling $t \mathrm{OHe}$ particles are effectively slowed down only after they fall down the terrestrial surface in $16 S_{2}$ meters of water (or $4 S_{2}$ meters of rock). Then they drift with velocity $V=\frac{g}{n \sigma v} \approx 8 S_{2} A^{1 / 2} \mathrm{~cm} / \mathrm{s}$ (where $A \sim 30$ is the average atomic weight in terrestrial surface matter, and $g=980 \mathrm{~cm} / \mathrm{s}^{2}$ ), sinking down the center of the Earth on a timescale $t=R_{E} / V \approx 1.5 \times 10^{7} S_{2}^{-1} \mathrm{~s}$, where $R_{E}$ is the radius of the Earth.

The in-falling techni-O-helium flux from the dark matter halo is $\mathcal{F}=n_{o} v_{h} / 8 \pi$, where the number density of $t \mathrm{OHe}$ in the vicinity of the solar system is $n_{o}=3 \times$ $10^{-3} S_{2}^{-1} \mathrm{~cm}^{-3}$ and the averaged velocity $v_{h} \approx$ $3 \times 10^{7} \mathrm{~cm} / \mathrm{s}$. During the lifetime of the Earth $\left(t_{E} \approx\right.$ $10^{17} \mathrm{~s}$ ), about $2 \cdot 10^{38} \mathrm{~S}_{2}^{-1}$ techni-O-helium atoms were captured. If $t \mathrm{OHe}$ dominantly sinks down the Earth, it should be concentrated near the Earth's center within a radius $R_{o c} \sim \sqrt{3 T_{c} /\left(m_{o} 4 \pi G \overline{\rho_{c}}\right)}$, which is $\leq 10^{8} S_{2}^{-1 / 2} \mathrm{~cm}$, for the Earth's central temperature $T_{c} \sim 10^{4} \mathrm{~K}$ and density $\rho_{c} \sim 4 \mathrm{~g} / \mathrm{cm}^{3}$.

Near the Earth's surface, the techni-O-helium abundance is determined by the equilibrium between the infalling and down-drifting fluxes. It gives

$$
n_{o E}=2 \pi \mathcal{F} / V=3 \times 10^{3} \cdot S_{2}^{-2} \cdot A^{-1 / 2} \mathrm{~cm}^{-3},
$$

or for $A \sim 30$ about $5 \times 10^{2} \cdot S_{2}^{-2} \mathrm{~cm}^{-3}$. This number density corresponds to the fraction

$$
f_{o E} \sim 5 \times 10^{-21} \cdot S_{2}^{-2}
$$

relative to the number density of the terrestrial atoms $n_{A} \approx$ $10^{23} \mathrm{~cm}^{-3}$.

These neutral $\left({ }^{4} \mathrm{He}^{++} A^{--}\right)$atoms may provide a catalysis of cold nuclear reactions in ordinary matter (much more effectively than muon catalysis). This effect needs a special and thorough investigation. On the other hand, if $A^{--}$ capture by nuclei, heavier than helium, is not effective and does not lead to a copious production of anomalous isotopes, the $\left({ }^{4} \mathrm{He}^{++} A^{--}\right)$diffusion in matter is determined by the elastic collision cross section (40) and may effectively hide techni-O-helium from observations.

One can give the following argument for an effective regeneration and quasielastic collisions of techni-O-helium in terrestrial matter. The techni-O-helium can be destroyed in the reactions (35). Then, free $A^{--}$are released and due to a hybrid Auger effect (capture of $A^{--}$, ejection of ordinary $e$ from the atom with atomic number $A$, and charge of the nucleus $Z$ ), $A^{--}$-atoms are formed, in which $A^{--}$occupies highly an excited level of the $\left.{ }_{Z}^{A} Q A\right)$ system, which is still much deeper than the lowest electronic shell of the considered atom. The $\left.{ }_{Z}^{A} Q A\right)$ atomic transitions to lower-lying states cause radiation in the intermediate range between atomic and nuclear transitions. In the course of this falling down to the center of the $\left(Z-A^{--}\right)$system, the nucleus approaches $A^{--}$. For $A>3$ the energy of the lowest state $n$ (given by $E_{n}=\frac{M \bar{\alpha}^{2}}{2 n^{2}}=\frac{2 A m_{p} Z^{2} \alpha^{2}}{n^{2}}$ ) of the ( $Z-$ $A^{--}$) system (having reduced mass $M \approx A m_{p}$ ) with a Bohr orbit $r_{n}=\frac{n}{M \bar{\alpha}}=\frac{n}{2 A Z m_{p} \alpha}$, exceeding the size of the nucleus $r_{A} \sim A^{1 / 3} m_{\pi}^{-1}$ ( $m_{\pi}$ being the mass of the pion), is less than the binding energy of $t \mathrm{OHe}$. Therefore the regeneration of techni-O-helium in a reaction, inverse to (35), takes place. An additional reason for the domination of the elastic channel of the reactions (35) is that the final state nucleus is created in the excited state and its deexcitation via $\alpha$-decay can also result in techni-O-helium regeneration. If regeneration is not effective and $A^{--}$remains bound to the heavy nucleus, anomalous isotope of $Z-2$ element should appear. This is a serious problem for the considered model.

However, if the general picture of sinking down is valid, it might give no more than the ratio $f_{o E} \sim 5 \times 10^{-21} \cdot S_{2}^{-2}$ of number density of anomalous isotopes to the number density of atoms of terrestrial matter around us, which is below the experimental upper limits for elements with $Z \geq$ 2. For comparison, the best upper limits on the anomalous helium were obtained in [53]. It was found, by searching with the use of laser spectroscopy for a heavy helium isotope in the Earth's atmosphere, that in the mass range $5 \mathrm{GeV}-10000 \mathrm{GeV}$, the terrestrial abundance (the ratio of anomalous helium number to the total number of atoms in the Earth) of anomalous helium is less than $2 \times 10^{-19}-3 \times$ $10^{-19}$. Since reactions induced by techni-O-helium lead to formation of anomalous isotopes with $Z \geq 2$, the results of ocean water experiments [54], putting severe constraints on anomalous hydrogen, are not appropriate to our case.

\section{Direct search for techni-O-helium}

It should be noted that the nuclear cross section of the techni-O-helium interaction with matter escapes the severe constraints [24] on strongly interacting dark matter particles [23,24] imposed by the XQC experiment [55].

In underground detectors, $t \mathrm{OHe}$ atoms are slowed down to thermal energies and give rise to energy transfer $\sim 2.5 \times$ $10^{-3} \mathrm{eVA} / S_{2}$, far below the threshold for direct dark matter detection. It makes this form of dark matter insensitive to the CDMS constraints. However, $t \mathrm{OHe}$ induced nuclear transformation can result in observable effects.

Therefore, a special strategy of such a search is needed that can exploit sensitive dark matter detectors on the ground or in space. In particular, as it was revealed in [56], a few $\mathrm{g}$ of superfluid ${ }^{3} \mathrm{He}$ detector [57] situated in a 
ground-based laboratory can be used to put constraints on the in-falling techni-O-helium flux from the galactic halo.

\section{DISCUSSION}

In this paper we explored the cosmological implications of a walking technicolor model with doubly charged technibaryons and technileptons. The considered model escapes most of the drastic problems of the sinister universe [9], related to the primordial ${ }^{4} \mathrm{He}$ cage for -1 charged particles and a consequent overproduction of anomalous hydrogen [10]. These charged ${ }^{4} \mathrm{He}$ cages pose a serious problem for composite dark matter models with single charged particles, since their Coulomb barrier prevents successful recombination of positively and negatively charged particles. The doubly charged $A^{--}$ techniparticles considered in this paper bind with ${ }^{4} \mathrm{He}$ in the techni-O-helium neutral states.

To avoid overproduction of anomalous isotopes, an excess of -2 charged techniparticles over their antiparticles should be generated in the Universe. In all the previous realizations of composite dark matter scenarios, this excess was put by hand to saturate the observed dark matter density. In our paradigm, this abundance of technibaryons and/or technileptons is connected naturally to the baryon relic density.

A challenging problem that we leave for future work is the nuclear transformations, catalyzed by techni-O-helium. The question about their consistency with observations remains open, since special nuclear physics analysis is needed to reveal what are the actual techni-O-helium effects in SBBN and in terrestrial matter. Another aspect of the considered approach is more clear. For reasonable values of the techniparticle mass, the amount of primordial ${ }^{4} \mathrm{He}$ bound in this atomlike state is significant and should be taken into account in comparison to observations.

The destruction of techni-O-helium by cosmic rays in the Galaxy releases free charged techniparticles, which can be accelerated and contribute to the flux of cosmic rays. In this context, the search for techniparticles at accelerators and in cosmic rays acquires the meaning of a crucial test for the existence of the basic components of the composite dark matter. At accelerators, techniparticles would look like stable doubly charged heavy leptons, while in cosmic rays, they represent a heavy -2 charge component with anomalously low ratio of electric charge to mass.

To conclude, walking technicolor cosmology can naturally resolve most of the problems of composite dark matter. Therefore, the model considered in this paper with stable -2 charged particles might provide a realistic physical basis for a composite dark matter scenario.

\section{ACKNOWLEDGMENTS}

The work of C.K. is supported by the Marie Curie Fellowship under Contract No. MEIF-CT-2006-039211. We are grateful to K.M. Belotsky and F. Sannino for reading the manuscript and for important remarks. M. $\mathrm{Kh}$. is grateful to CERN (Geneve, Switzerland) and to ICTP (Trieste, Italy) for hospitality.

\section{APPENDIX A: CHARGE ASYMMETRY IN FREEZING OUT OF PARTICLES AND ANTIPARTICLES}

The frozen number density of cosmic relics, which were in equilibrium with the primordial plasma, is conventionally deduced ${ }^{2}$ from the equation [58]

$$
\dot{n}+3 H n=\left\langle\sigma_{\mathrm{ann}} v\right\rangle\left(n_{\text {eq }}^{2}-n^{2}\right) .
$$

This equation is written for the case of a charge symmetry of the particles in question, i.e., for the case when number densities of particles $X$ and antiparticles $\bar{X}$ are equal $n_{X}=$ $n_{\bar{X}}=n$. The value $n_{\text {eq }}$ corresponds to their equilibrium number density and is given by the Boltzmann distribution

$$
n_{\text {eq }}=g_{S}\left(\frac{m T}{2 \pi}\right)^{3 / 2} \exp \left(-\frac{m}{T}\right)
$$

Here $g_{S}$ and $m$ are the number of spin states and the mass of the given particle.

During the cooling, $n_{\text {eq }}$ decreases exponentially and becomes, below the freezing out temperature $T_{f}$, much smaller than the real density $n$, so the term $\left\langle\sigma_{\mathrm{ann}} v\right\rangle n_{\mathrm{eq}}^{2}$, describing the creation of $X \bar{X}$ from the plasma can be neglected [59]. It allows us to obtain an approximate solution of Eq. (A1).

In case of a charge asymmetry one needs to split Eq. (A1) in two: for $n_{X}$ and $n_{\bar{X}}$, which are not equal now.

$$
\begin{aligned}
& \dot{n}_{X}+3 H n_{X}=\left\langle\sigma_{\mathrm{ann}} v\right\rangle\left(n_{\mathrm{eq} X} n_{\mathrm{eq} \bar{X}}-n_{X} n_{\bar{X}}\right), \\
& \dot{n}_{\bar{X}}+3 H n_{\bar{X}}=\left\langle\sigma_{\mathrm{ann}} v\right\rangle\left(n_{\mathrm{eq} X} n_{\mathrm{eq} \bar{X}}-n_{X} n_{\bar{X}}\right) .
\end{aligned}
$$

The values $n_{\mathrm{eq} X}$ and $n_{\mathrm{eq} \bar{X}}$ are given by Eq. (A2) with inclusion of the chemical potential, which for $X$ and for $\bar{X}$ are related as $\mu_{X}=-\mu_{\bar{X}}=\mu$ (see, e.g., [60]). So

$$
n_{\text {eq } X, \bar{X}}=\exp \left( \pm \frac{\mu}{T}\right) n_{\text {eq }}
$$

where the upper and lower signs are for $X$ and $\bar{X}$, respectively. So

$$
n_{\mathrm{eq} X} n_{\mathrm{eq} \bar{X}}=n_{\mathrm{eq}}^{2} .
$$

A degree of asymmetry will be described in the conventional manner (as for baryons) by the ratio of the difference between $n_{X}$ and $n_{\bar{X}}$ to the number density of relic photons at the modern period

$$
\kappa_{\gamma \bmod }=\frac{n_{X \bmod }-n_{\bar{X} \bmod }}{n_{\gamma \bmod }} .
$$

\footnotetext{
${ }^{2}$ We follow here the results obtained in $[10,13]$ with the help of K. M. Belotsky
} 
However, for practical purposes it is more suitable to use the ratio to the entropy density, which unlike Eq. (A6), does not change in time provided entropy conservation. The photon number density $n_{\gamma}$ and the entropy density $s$ are given by

$$
n_{\gamma}=\frac{2 \zeta(3)}{\pi^{2}} T^{3}, \quad s=\frac{2 \pi^{2} g_{s}}{45} T^{3}=1.80 g_{s} n_{\gamma},
$$

where

$$
g_{s}=\sum_{\mathrm{bos}} g_{S}\left(\frac{T_{\mathrm{bos}}}{T}\right)^{3}+\frac{7}{8} \sum_{\text {ferm }} g_{S}\left(\frac{T_{\text {ferm }}}{T}\right)^{3} .
$$

The sums in Eq. (A8) are over ultrarelativistic bosons and fermions. So

$$
\kappa=\frac{n_{X}-n_{\bar{X}}}{s}, \quad \kappa=\frac{\kappa_{\gamma \bmod }}{1.8 g_{s \bmod }},
$$

where $g_{s \text { mod }}=43 / 11 \approx 3.91$. Equation (A9) provides a connection between $n_{X}$ and $n_{\bar{X}}$. Let us pass to the variables

$$
r_{+}=\frac{n_{X}}{s}, \quad r_{-}=\frac{n_{\bar{X}}}{s}, \quad r=\frac{n_{X}+n_{\bar{X}}}{s}, \quad x=\frac{T}{m} .
$$

The apparent relations between the $r_{i}$ are

$$
r_{+}-r_{-}=\kappa, \quad r_{+}+r_{-}=r .
$$

Provided that the essential entropy redistribution does not take place ( $g_{s}=$ const) during the period of freezing out, a transformation to the variable $x$ is possible:

$$
-H d t=d T / T=d x / x .
$$

On the RD stage the Hubble parameter depends on $T$ as

$$
H=\frac{2 \pi}{3} \sqrt{\frac{\pi g_{\epsilon}}{5}} \frac{T^{2}}{m_{P l}}
$$

where $g_{\epsilon}$ is given by

$$
g_{\epsilon}=\sum_{\text {bos }} g_{S}\left(\frac{T_{\text {bos }}}{T}\right)^{4}+\frac{7}{8} \sum_{\text {ferm }} g_{S}\left(\frac{T_{\text {ferm }}}{T}\right)^{4} .
$$

For $r_{+}, r_{-}$, and $r$ from Eqs. (A3) one obtains the equations

$$
\begin{aligned}
\frac{d r_{+}}{d x} & =f_{1}\left\langle\sigma_{\mathrm{ann}} v\right\rangle\left(r_{+}\left(r_{+}-\kappa\right)-f_{2}(x)\right), \\
\frac{d r_{-}}{d x} & =f_{1}\left\langle\sigma_{\mathrm{ann}} v\right\rangle\left(r_{-}\left(r_{-}+\kappa\right)-f_{2}(x)\right), \\
\frac{d r}{d x} & =\frac{1}{2} f_{1}\left\langle\sigma_{\mathrm{ann}} v\right\rangle\left(r^{2}-\kappa^{2}-4 f_{2}(x)\right) .
\end{aligned}
$$

Here

$$
f_{1}=\frac{s}{H x}, \quad f_{2}(x)=\frac{n_{\mathrm{eq}}^{2}}{s^{2}}=\frac{45^{2} g_{S}^{2}}{2^{5} \pi^{7} g_{s}^{2} x^{3}} \exp \left(-\frac{2}{x}\right) .
$$

By using Eqs. (A7) and (A12), one finds that on the RD stage $f_{1}$ is

$$
f_{1}=\sqrt{\frac{\pi g_{s}^{2}}{45 g_{\epsilon}}} m_{P l} m
$$

and independent of $x$.

To solve Eqs. (A14) analogously to Eq. (A1), namely, neglecting $f_{2}(x)$ in them, starting with some $x=x_{f}$, it would not be more difficult to define the moment $x=x_{f}$. Nonetheless, if one supposes that such a moment is defined, $r_{i}$ will be

$$
\begin{aligned}
r_{+}(x \approx 0) & =\frac{\kappa \cdot r_{+f}}{r_{+f}-\left(r_{+f}-\kappa\right) \exp (-\kappa J)}, \\
r_{-}(x \approx 0) & =\frac{\kappa \cdot r_{-f}}{\left(\kappa+r_{-f}\right) \exp (\kappa J)-r_{-f}}, \\
r(x \approx 0) & =\kappa \frac{\left(\kappa+r_{f}\right) \exp (\kappa J)+r_{f}-\kappa}{\left(\kappa+r_{f}\right) \exp (\kappa J)-\left(r_{f}-\kappa\right)} .
\end{aligned}
$$

Here $r_{i f}=r_{i}\left(x=x_{f}\right)$,

$$
J=\int_{0}^{x_{f}} f_{1}\left\langle\sigma_{\mathrm{ann}} v\right\rangle d x
$$

All $r_{i}$ (at any moment) are related with the help of Eqs. (A11). Taking into account Eq. (A4) or Eq. (A5) for $r_{i f}$ one obtains

$$
r_{ \pm f}=\frac{1}{2}\left(\sqrt{4 f_{2}\left(x_{f}\right)+\kappa^{2}} \pm \kappa\right), \quad r_{f}=\sqrt{4 f_{2}\left(x_{f}\right)+\kappa^{2}}
$$

For $\left\langle\sigma_{\mathrm{ann}} v\right\rangle$ independent of $x$ on the RD stage, $f_{1}$ also independent of $x$, and $x_{f}$ defined from the condition $R\left(T_{f}\right)=H\left(T_{f}\right)$ for the reaction rate $R\left(T_{f}\right)=n_{\text {eq }}\left(T_{f}\right) \times$ $\left\langle\sigma_{\mathrm{ann}} v\left(T_{f}\right)\right\rangle$, leading to

$$
\begin{aligned}
n_{\mathrm{eq}}\left(T_{f}\right)\left\langle\sigma_{\mathrm{ann}} v\left(T_{f}\right)\right\rangle / H\left(T_{f}\right) & =\frac{n_{\mathrm{eq}}}{s} \cdot \frac{s}{H x_{f}} \cdot\left\langle\sigma_{\mathrm{ann}} v\left(x_{f}\right)\right\rangle \cdot x_{f} \\
& =\sqrt{f_{2}\left(x_{f}\right)} f_{1}\left\langle\sigma_{\mathrm{ann}} v\left(x_{f}\right)\right\rangle \cdot x_{f} \\
& =1,
\end{aligned}
$$

one obtains

$$
\sqrt{f_{2}\left(x_{f}\right)}=\frac{1}{f_{1}\left\langle\sigma_{\mathrm{ann}} v\right\rangle \cdot x_{f}}=\frac{1}{J} .
$$

If (a) $\left\langle\sigma_{\text {ann }} v\right\rangle=\alpha^{2} / m^{2}$ or (b) $\left\langle\sigma_{\text {ann }} v\right\rangle=\alpha / \sqrt{T m^{3}}$ and one assumes $f_{1}=$ const, then

$$
J_{a}=\sqrt{\frac{\pi g_{s}^{2}}{45 g_{\epsilon}}} m_{P l} \frac{\alpha^{2}}{m} x_{f}, \quad J_{b}=\sqrt{\frac{\pi g_{s}^{2}}{45 g_{\epsilon}}} m_{P l} \frac{\alpha}{m} 2 \sqrt{x_{f}} .
$$




\section{APPENDIX B: PRIMORDIAL TECHNILEPTONS FROM THE BIG BANG UNIVERSE}

As already mentioned, the minimal walking technicolor model considered in this paper can allow the creation of $\zeta$ excess that might contribute (or even saturate) to the modern dark matter density in the form of techni-O-helium atoms. For light baryon excess $\eta_{B}=n_{B \text { mod }} / n_{\gamma \bmod }=6 \times$ $10^{-10}$, it gives a $\zeta$-excess

$$
\eta_{\zeta}=n_{\zeta \text { mod }} / n_{\gamma \bmod }=3 \times 10^{-11} f_{\zeta}\left(\frac{100 \mathrm{GeV}}{m_{\zeta}}\right),
$$

where $m_{\zeta}$ is the mass of $\zeta$. By definition $f_{\zeta}$ is the contribution of $\zeta$ into the modern dark matter density $f_{\zeta}=1-$ $x$, where $x$ was defined in Eq. (18). For future use, following $[9,10]$, it is convenient to relate the baryon density $\Omega_{B}=0.044$ and the technilepton density $\Omega_{L^{\prime}}=0.224$ with the entropy density $s$, and to introduce $r_{B}=n_{B} / s$ and $r_{\zeta}=n_{\zeta} / s$. Taking into account that $s_{\text {mod }}=$ $7.04 \cdot n_{\gamma \text { mod }}$, one obtains $r_{B} \sim 8 \cdot 10^{-11}$ and

$$
r_{\zeta}=4 \cdot 10^{-12}\left(\frac{100 \mathrm{GeV}}{m_{\zeta}}\right)=4 \times 10^{-12} f_{\zeta} / S_{2} .
$$

\section{Chronological cornerstones of the technilepton Universe}

After the generation of technilepton asymmetry, the thermal history of technileptons in chronological order looks as follows:

(1) $10^{-10} S_{2}^{-2} \mathrm{~s} \leq t \leq 6 \times 10^{-8} S_{2}^{-2} \mathrm{~s}$ at $m_{\zeta} \geq T \geq$ $T_{f}=m_{\zeta} / 31 \approx 3 S_{2} \mathrm{GeV}$. $\zeta$-lepton pair $\zeta \bar{\zeta}$ annihilation and freezing out. For large $m_{\zeta}$ the abundance of frozen out $\zeta$-lepton pairs is not suppressed in spite of an $\zeta$-lepton excess.

(2) $t \sim 2.4 \times 10^{-3} S_{2}^{-2} \mathrm{~s}$ at $T \sim I_{\zeta}=20 S_{2} \mathrm{MeV}$. The temperature corresponds to the binding energy $I_{\zeta}=$ $Z_{\zeta}^{4} \alpha^{2} m_{\zeta} / 4 \approx 20 S_{2} \mathrm{MeV} \quad\left(Z_{\zeta}=2\right) \quad$ of $\zeta$-positronium atoms $\left(\zeta^{--} \bar{\zeta}^{++}\right)$, in which $\bar{\zeta}^{++}$annihilate. At large $m_{\zeta}$ this annihilation is not at all effective to reduce the $\zeta \bar{\zeta}$ pairs abundance. These pairs are eliminated in the course of the successive evolution of $\zeta$-matter.

(3) $100 \mathrm{~s} \leq t \leq 300 \mathrm{~s}$ at $100 \mathrm{keV} \geq T \geq I_{o} / 27 \approx$ $60 \mathrm{keV} .{ }^{4} \mathrm{He}$ is formed in the SBBN and virtually all free $\zeta^{--}$are trapped by ${ }^{4} \mathrm{He}$ in $\left({ }^{4} \mathrm{He}^{++} \zeta^{--}\right)$. Note that in the period $100 \mathrm{keV} \leq T \leq 1.6 \mathrm{MeV}$, ${ }^{4} \mathrm{He}$ is not formed, therefore it is only after the first three minutes, when $\left({ }^{4} \mathrm{He}^{++} \zeta^{--}\right)$trapping of $\zeta^{--}$ can take place. Being formed, techni-OLe-helium catalyzes the binding of free $\bar{\zeta}^{++}$with its constituent $\zeta^{--}$into $\zeta$-positronium and complete annihilation of all the primordial antiparticles. At large $m_{\zeta}$, effects of $\left(\zeta^{--} \bar{\zeta}^{++}\right)$annihilation, catalyzed by techni-O-helium, do not cause any contradictions with observations.
(4) $t \sim 10^{12} \mathrm{~s}$ at $T \sim T_{\mathrm{RM}} \approx 1 \mathrm{eV}$. The techniparticle dominance starts with techni-O-helium atoms playing the role more close to warm dark matter in the formation of large scale structures.

\section{Freezing out of $\zeta$-leptons}

In the early Universe at temperatures highly above their masses, the $\zeta$-fermions were in thermodynamical equilibrium with the relativistic plasma. It means that at $T>m_{\zeta}$ the excessive $\zeta$ were accompanied by $\zeta \bar{\zeta}$ pairs.

During the expansion, when the temperature $T$ falls below the mass of the $\zeta$-particles, the concentration of particles and antiparticles is given by the equilibrium. The equilibrium concentration of $\zeta \bar{\zeta}$ pairs starts to decrease at $T<m_{\zeta}=100 S_{2} \mathrm{GeV}$. At the freezing out temperature $T_{f}$, the rate of expansion exceeds the rate of annihilation to photons $\zeta \bar{\zeta} \rightarrow \gamma \gamma$, to $W, Z$-bosons $\zeta \bar{\zeta} \rightarrow$ $W W(Z Z)$, or to pairs of light fermions $f$ (quarks and charged leptons) $\zeta \bar{\zeta} \rightarrow \bar{f} f$ (the latter takes place both due to electromagnetic and weak interactions). Then $\zeta$ leptons and their antiparticles $\bar{\zeta}$ are frozen out.

In the case of freezing out of $\zeta$-leptons one has (see Appendix A)

$$
f_{1 \zeta}=\sqrt{\frac{\pi g_{s}^{2}}{45 g_{\epsilon}}} m_{P l} m_{\zeta} \approx 2.5 m_{P l} m_{\zeta},
$$

$\left\langle\sigma_{\mathrm{ann}} v\right\rangle=\frac{\bar{\alpha}^{2}}{m_{\zeta}^{2}}$, and

$$
J_{\zeta}=\sqrt{\frac{\pi g_{s}^{2}}{45 g_{\epsilon}}} m_{P l} \frac{\bar{\alpha}^{2}}{m_{\zeta}} x_{f},
$$

where $\bar{\alpha}=Z_{\zeta}^{2} \alpha+\bar{\alpha}_{e w}, Z_{\zeta}=2$ is the absolute value of the electric charge of the $\zeta$ and $\bar{\alpha}_{e w}$ takes into account the effects of $W$ and $Z$ bosons in technilepton annihilation. By putting in Eq. (A15) $g_{S}=2, g_{s} \sim 100$, one obtains the solution of the transcendent Eq. (A19)

$$
\begin{aligned}
x_{f} & \approx\left(\ln \left(\frac{45 g_{S}}{2^{5 / 2} \pi^{7 / 2} g_{s}} \cdot f_{1 \zeta}\left\langle\sigma_{\mathrm{ann}} v\right\rangle\right)\right)^{-1} \\
& \approx \frac{1}{30} \cdot \frac{1}{\left(1-\ln \left(S_{2}\right) / 30\right)} .
\end{aligned}
$$

Taking $g_{s} \approx g_{\epsilon} \sim 100$, one finds from Eq. (A13) $J_{\zeta}=$ $6.5 \times 10^{13} / S_{2}\left(1-\ln \left(S_{6}\right) / 30\right)^{-1}$ and from Eq. (A19) $\sqrt{4 f_{2}\left(x_{f}\right)}=2 / J_{\zeta}=3 \times 10^{-13} S_{2} \cdot\left(1-\ln \left(S_{2}\right) / 30\right)$. For $\kappa=r_{\zeta}=4 \times 10^{-12} f_{\zeta} / S_{2}$, one has $\kappa J_{\zeta}=26 f_{\zeta} / S_{2}^{2}$. At $S_{2}<2.74 f_{2}\left(x_{f}\right)<\kappa^{2}$ and $r_{ \pm f}$ is given by Eq. (A17). Since $4 f_{2}\left(x_{f}\right) \gg \kappa^{2}$ for $S_{2} \gg 1$, one obtains from Eq. (A17)

$$
r_{ \pm f}=\frac{1}{2}\left(\sqrt{4 f_{2}\left(x_{f}\right)} \pm \kappa\right) .
$$

The frozen out abundances of $\zeta$-leptons and their antiparticles are given by 


$$
\begin{aligned}
& r_{\zeta}=\frac{\kappa \cdot r_{+f}}{r_{+f}-\left(r_{+f}-\kappa\right) \exp \left(-\kappa J_{\zeta}\right)}=F_{\zeta}\left(S_{2}\right), \\
& r_{\bar{\zeta}}=\frac{\kappa \cdot r_{-f}}{\left(\kappa+r_{-f}\right) \exp \left(\kappa J_{\zeta}\right)-r_{-f}}=F_{\bar{\zeta}}\left(S_{2}\right) .
\end{aligned}
$$

For growing $S_{2} \gg 1$, the solution Eq. (B5) approaches the values

$$
\begin{aligned}
r_{\zeta} & \approx \sqrt{f_{2}\left(x_{f}\right)}+\kappa / 2 \\
& \approx 1.5 \times 10^{-13} S_{2} \cdot\left(1-\ln \left(S_{2}\right) / 30\right)+2 \times 10^{-12} f_{\zeta} / S_{2}, \\
r_{\bar{\zeta}} & \approx \sqrt{f_{2}\left(x_{f}\right)}-\kappa / 2 \\
& \approx 1.5 \times 10^{-13} S_{2} \cdot\left(1-\ln \left(S_{2}\right) / 30\right)-2 \times 10^{-12} f_{\zeta} / S_{2} .
\end{aligned}
$$

At $S_{2}<5 f_{\zeta}$, the factor in the exponent $\kappa J_{\zeta}$ exceeds 1 , and some suppression of the $(\bar{\zeta})$-abundance takes place. For $S_{2}$ close to 1 , one has

$$
\begin{aligned}
& r_{\zeta}=F_{\zeta}\left(S_{2}\right) \approx \kappa=4 \times 10^{-12} f_{\zeta} / S_{2}, \\
& r_{\bar{\zeta}}=F_{\bar{\zeta}}\left(S_{2}\right) \approx 5 \times 10^{-3} \kappa S_{2}^{4} \exp \left(-26 f_{\zeta} / S_{2}^{2}\right) .
\end{aligned}
$$

At $S_{2}=1$, the factor in the exponent reaches the value $\kappa J_{\zeta}=26 f_{\zeta}$ and the solution Eq. (B5) gives $r_{\zeta} \approx \kappa=4 \times$ $10^{-12} f_{\zeta}$ and

$$
\begin{aligned}
r_{\bar{\zeta}} & \approx \frac{\kappa \cdot r_{-f}}{\kappa+r_{-f}} \exp \left(-\kappa J_{\zeta}\right) \approx r_{-f} \exp \left(-\kappa J_{\zeta}\right) \\
& \approx 10^{-14} \exp \left(-26 f_{\zeta}\right) \approx 3 \times 10^{-25}
\end{aligned}
$$

for $f_{\zeta}=1$ and $r_{-f} \approx 10^{-14}$ from Eq. (A17).

The $S_{2}$-dependence of the frozen out abundances (in units of the entropy density) of the $\zeta$ leptons and their antiparticles are

$$
r_{\zeta}=F_{\zeta}\left(S_{2}\right), \quad r_{\bar{\zeta}}=F_{\bar{\zeta}}\left(S_{2}\right)
$$

given by Eq. (B5). For growing $S_{2} \gg 1$, the solution Eq. (B5) approaches the values

$$
\begin{aligned}
r_{A C} \approx & \sqrt{f_{2}\left(x_{f}\right)}+\kappa / 2 \\
\approx & 1.5 \times 10^{-13} S_{2} \cdot\left(1-\ln \left(S_{2}\right) / 30\right)+2 \\
& \times 10^{-12} f_{\zeta} / S_{2}, \\
r_{A C} \approx & \sqrt{f_{2}\left(x_{f}\right)}-\kappa / 2 \\
\approx & 1.5 \times 10^{-13} S_{2} \cdot\left(1-\ln \left(S_{2}\right) / 30\right)-2 \\
& \times 10^{-12} f_{\zeta} / S_{2} .
\end{aligned}
$$

At $S_{2}<5 f_{\zeta}$, there is a exponential suppression of the $\bar{\zeta}$ abundance. For $S_{2}$ close to 1, one has

$$
\begin{aligned}
& r_{\zeta}=F_{\zeta}\left(S_{2}\right) \approx \kappa=4 \times 10^{-12} / S_{2}, \\
& r_{\bar{\zeta}}=f_{\bar{\zeta}}\left(S_{2}\right) \approx 5 \times 10^{-3} \kappa S_{2}^{4} \exp \left(-26 f_{\zeta} / S_{2}^{2}\right) .
\end{aligned}
$$

At $S_{2}=1$, the solution Eq. (B10) gives

$$
r_{\zeta} \approx \kappa_{A}=\kappa_{C}=2 \times 10^{-12} f_{\zeta},
$$

and

$$
r_{\bar{\zeta}} \approx 3 \times 10^{-25}
$$

On the other hand at $S_{2}>5$, the concentration of frozen out $\zeta$-lepton pairs exceeds the one of the $\zeta$-lepton excess given by Eq. (B2) and this effect grows with $S_{2}$ as $\propto S_{2}^{2}$ at large $S_{2}$. So in this moment, in spite of an assumed $\zeta$-lepton asymmetry, the frozen out concentration of antiparticles $\bar{\zeta}$ is not strongly suppressed and they cannot be neglected in the cosmological evolution of $\zeta$-matter.

The antiparticles $\bar{\zeta}$ should be effectively annihilated in the successive processes of $\zeta \bar{\zeta}$ recombination in bound $(\zeta \bar{\zeta}) \zeta$-positronium states.

\section{3. $\bar{\zeta}$ annihilation in $\zeta$-positronium states}

The frozen out antiparticles $\bar{\zeta}$ can bind at $T<I_{\zeta}$ with the corresponding particles $\zeta$ into positroniumlike systems and annihilate. The binding is provided by the Coulomb interaction of electromagnetic charges $Z_{\zeta}=2$. Since the lifetime of these positroniumlike systems is much less than the timescale of their disruption by energetic photons, the direct channel of $\bar{\zeta}$ and $\bar{\zeta}$ binding in $(\zeta \bar{\zeta})$, followed by a rapid annihilation, cannot be compensated by an inverse reaction of photo-ionization. That is why $\bar{\zeta}$ begins to bind with $\zeta$ and annihilates as soon as the temperature becomes less than $I_{\zeta}$. The decrease of the $\bar{\zeta}$ abundance due to the $\zeta \bar{\zeta}$ recombination is governed by the equation

$$
\frac{d r_{\bar{\zeta}}}{d t}=-r_{\zeta} r_{\bar{\zeta}} \cdot s \cdot\langle\sigma v\rangle,
$$

where $s$ is the entropy density and

$$
\langle\sigma v\rangle=\left(\frac{16 \pi}{3^{3 / 2}}\right) \cdot \frac{\bar{\alpha}}{T^{1 / 2} \cdot m_{\zeta}^{3 / 2}} .
$$

Here $\bar{\alpha}=Z_{\zeta}^{2} \alpha$.

In the analysis of various recombination processes, we can use the interpolation formula for the recombination cross section deduced in $[3,10,13]$ :

$$
\sigma_{r}=\left(\frac{2 \pi}{3^{3 / 2}}\right) \cdot \frac{\bar{\alpha}^{3}}{T \cdot I_{1}} \cdot \log \left(\frac{I_{1}}{T}\right)
$$

and the recombination rate given by $[3,10]$

$$
\langle\sigma v\rangle=\left(\frac{2 \pi}{3^{5 / 2}}\right) \cdot \frac{\bar{\alpha}^{3}}{T \cdot I_{1}} \cdot \log \left(\frac{I_{1}}{T}\right) \cdot \frac{k_{\text {in }}}{M} .
$$

Here $k_{\text {in }}=\sqrt{2 T M}, I_{1} \approx \bar{\alpha}^{2} M / 2$ is the ionization potential, and $M$ is the reduced mass for a pair of recombining particles. The constant $\bar{\alpha}$ for recombining particles with charges $Z_{1}$ and $Z_{2}$ is related to the fine structure constant $\alpha$ via $\bar{\alpha}=Z_{1} Z_{2} \alpha$. The approximation Eq. (B13) followed 
from the known result for electron-proton recombination

$$
\sigma_{\mathrm{rec}}=\sigma_{r}=\sum_{i} \frac{8 \pi}{3^{3 / 2}} \bar{\alpha}^{3} \frac{e^{4}}{M v^{2} i^{3}} \frac{1}{\left(M v^{2} / 2+I_{i}\right)},
$$

where $v$ is the velocity of the particles. $I_{i}$ is the ionization potential $\left(I_{i}=I_{1} / i^{2}\right)$. The index $i$ runs from one to infinity.

To sum approximately over $i$, it was noted in $[3,13]$ that $\sigma_{r} \propto 1 / i$ for $I_{i} \gg M v^{2} / 2=T_{\text {eff }}$, while at $I_{i}<T_{\text {eff }}$, the cross section $\sigma_{i} \propto 1 / i^{3}$ falls down rapidly.

Using the formalism of Appendix A, we can rewrite Eq. (B11) as

$$
\frac{d r_{\bar{\zeta}}}{d x}=f_{1 \bar{\zeta}}\langle\sigma v\rangle r_{\bar{\zeta}}\left(r_{\bar{\zeta}}+\kappa\right),
$$

where $x=T / I_{\zeta}$, the asymmetry $\kappa=r_{\zeta}-r_{\bar{\zeta}}=$ $4 \times 10^{-12} f_{\zeta} / S_{2}$ is given by Eq. (B2) and

$$
f_{1 \bar{\zeta}}=\sqrt{\frac{\pi g_{s}^{2}}{45 g_{\epsilon}}} m_{P l} I_{\zeta} \approx m_{P l} I_{\zeta} .
$$

The concentration of the remaining $\bar{\zeta}$ is given by Eq. (A17) of Appendix A

$$
r_{\bar{\zeta}}=\frac{\kappa \cdot r_{f \bar{\zeta}}}{\left(\kappa+r_{f \bar{\zeta}}\right) \exp \left(\kappa J_{\zeta}\right)-r_{f \bar{\zeta}}},
$$

where $r_{f \bar{\zeta}}$ is given by Eq. (B8) and

$$
\begin{aligned}
J_{\zeta} & =\int_{0}^{x_{f \bar{\zeta}}} f_{1 \bar{\zeta}}\langle\sigma v\rangle d x=m_{P l} I_{\zeta} 4 \pi\left(\frac{2}{3^{3 / 2}}\right) \cdot \frac{\bar{\alpha}^{2}}{I_{\zeta} \cdot m_{\zeta}} \cdot 2 \cdot x_{f \bar{\zeta}}^{1 / 2} \\
& \approx 0.8 \times 10^{15} / S_{2} .
\end{aligned}
$$

In the evaluation of Eq. (B17) we took into account that the decrease of $\bar{\zeta}$ starts at $T \sim I_{\zeta}$, so that $x_{f \bar{\zeta}} \sim 1$. At $S_{2}<$ $57 f_{\bar{\zeta}}^{1 / 2}$, the abundance of $\bar{\zeta}$ is suppressed exponentially.

Indeed, one has $\kappa J_{\zeta} \approx 3200 f_{\zeta} / S_{2}^{2}$ in the exponent of Eq. (B16). Similar to the case of the AC-leptons [13], it differs significantly from the situation revealed in [10] for the tera-positrons in Glashow's sinister model [9]. Though in both cases a decrease of antiparticles due to the formation of positroniumlike systems is induced by electromagnetic interaction and the factor in the exponent is determined by the square of the fine structure constant $\alpha$, in the case of $\zeta$-leptons, this factor is enhanced by $Z_{\zeta}^{4}=16$ times due to the $Z_{\zeta}^{4}$ dependence of $\bar{\alpha}^{2}$. It results in a much wider mass interval for $\zeta$-leptons, in which the primordial pair abundance is exponentially suppressed.

At $S_{2}$ close to 1 , the condition $r_{f \bar{\zeta}} \ll \kappa$ in the solution Eq. (B16) provides the approximate solution

$$
r_{\bar{\zeta}}=r_{f \bar{\zeta}} \cdot \exp \left(-\kappa J_{\zeta}\right) \approx 10^{-14} S_{2}^{3} \exp \left(-3200 f_{\zeta} / S_{2}^{2}\right) .
$$

For $S_{2}>5$, the condition $r_{f \bar{\zeta}} \gg \kappa$ is valid. Therefore the solution Eq. (B16) has the form

$$
r_{\bar{\zeta}} \approx \frac{\kappa}{\exp \left(\kappa J_{\zeta}\right)-1},
$$

which gives for $S_{2}<57 f_{\zeta}^{1 / 2}$

$$
r_{\bar{\zeta}}=\kappa \cdot \exp \left(-\kappa J_{\zeta}\right) \approx\left(\frac{2 \cdot 10^{-12}}{S_{2}}\right) \exp \left(-3200 f_{\zeta} / S_{2}^{2}\right) .
$$

At large $S_{2}>57 f_{\zeta}^{1 / 2}$, the approximate solution is given by

$$
r_{\bar{\zeta}} \approx \frac{1}{J_{\zeta}}-\frac{\kappa}{2} \approx 1.25 \times 10^{-15} S_{2}-2 \times 10^{-12} f_{\zeta} / S_{2} .
$$

In the result, the residual amount of $\bar{\zeta}$ remains at $S_{2}>$ $57 f_{\zeta}^{1 / 2}$ enormously high, being for $S_{2}>70 f_{\zeta}^{1 / 2}$ larger than the AC-lepton excess. This effect grows with $S_{2}>70 f_{\zeta}^{1 / 2}$ as $\propto S_{2}^{2}$.

The general expression for the $\zeta$ abundance $r_{\zeta}$ after the $\zeta$-positronium annihilation has the form [see Eq. (A17) of Appendix A]

$$
r_{\zeta}=\frac{\kappa \cdot r_{\zeta f}}{r_{\zeta f}-\left(r_{\zeta f}-\kappa\right) \exp \left(-\kappa J_{\zeta}\right)},
$$

where $J_{\zeta}$ is given by Eq. (B17) and $r_{\zeta f}$ is given by Eq. (B8). With the account for $r_{\zeta f}>\kappa$, for all $S_{2}$, one obtains

$$
r_{\zeta}=\frac{\kappa}{1-\exp \left(-\kappa J_{\zeta}\right)}
$$

This gives $r_{\zeta} \approx 1 / J_{\zeta}+\kappa / 2 \approx 3 \times 10^{-16} S_{2}+2 \times$ $10^{-12} f_{\zeta} / S_{2}$ for large $S_{2}$, and $\kappa$ for $S_{2}<57 f_{\zeta}^{1 / 2}$.
[1] D. Fargion et al., JETP Lett. 69, 434 (1999); K. M. Belotsky, M. Yu. Khlopov, and K. I. Shibaev, Gravitation and Cosmology 6, 140 (2000); K. M. Belotsky, D. Fargion, M. Yu. Khlopov, and R. Konoplich, Phys. At. Nucl. 71, 147 (2008); K. M. Belotsky, D. Fargion, M. Yu. Khlopov, R. Konoplich, and K.I. Shibaev, Gravitation and Cosmology 11, 16 (2005) and references therein.
[2] K. M. Belotsky, M. Yu. Khlopov, S. V. Legonkov, and K. I. Shibaev, Gravitation and Cosmology 11, 27 (2005).

[3] K. M. Belotsky, D. Fargion, M. Yu. Khlopov, R. Konoplich, M. G. Ryskin, and K. I. Shibaev, Gravitation and Cosmology 11, 3 (2005).

[4] M. Maltoni et al., Phys. Lett. B 476, 107 (2000); V. A. Ilyin et al., Phys. Lett. B 503, 126 (2001); V. A. Novikov 
et al., Phys. Lett. B 529, 111 (2002); JETP Lett. 76, 127 (2002).

[5] M. Yu. Khlopov, JETP Lett. 83, 1 (2006) [Pis'ma Zh. Eksp. Teor. Fiz. 83, 3 (2006)];

[6] K. Belotsky, M. Khlopov, and K. Shibaev, arXiv:astro-ph/ 0602261.

[7] K. Belotsky, M. Khlopov, and K. Shibaev, Gravitation and Cosmology 12, 1 (2006).

[8] M. Y. Khlopov, arXiv:astro-ph/0607048.

[9] S. L. Glashow, arXiv:hep-ph/0504287.

[10] D. Fargion and M. Khlopov, arXiv:hep-ph/0507087.

[11] C. A. Stephan, J. Phys. A 39, 9657 (2006).

[12] A. Connes, Noncommutative Geometry (Academic Press, London and San Diego, 1994).

[13] D. Fargion, M. Khlopov, and C. A. Stephan, Classical Quantum Gravity 23, 7305 (2006); arXiv:astro-ph/ 0511789.

[14] M.Y. Khlopov and C.A. Stephan, arXiv:astro-ph/ 0603187.

[15] F. Sannino and K. Tuominen, Phys. Rev. D 71, 051901 (2005); arXiv:hep-ph/0405209.

[16] D. K. Hong, S. D. H. Hsu, and F. Sannino, Phys. Lett. B 597, 89 (2004).

[17] D. D. Dietrich, F. Sannino, and K. Tuominen, Phys. Rev. D 72, 055001 (2005).

[18] D. D. Dietrich, F. Sannino, and K. Tuominen, Phys. Rev. D 73, 037701 (2006).

[19] S. B. Gudnason, C. Kouvaris, and F. Sannino, Phys. Rev. D 73, 115003 (2006).

[20] S. B. Gudnason, C. Kouvaris, and F. Sannino, Phys. Rev. D 74, 095008 (2006).

[21] V. K. Dubrovich and M. Y. Khlopov, JETP Lett. 77, 335 (2003) [Pis'ma Zh. Eksp. Teor. Fiz. 77, 403 (2003)]; arXiv:astro-ph/0206138; V.K. Dubrovich, D. Fargion, and M. Y. Khlopov, Astropart. Phys. 22, 183 (2004); arXiv:hep-ph/0312105; Nucl. Phys. B, Proc. Suppl. 136, 362 (2004).

[22] S. Dimopoulos et al., Phys. Rev. D 41, 2388 (1990).

[23] C. B. Dover, T. K. Gaisser, and G. Steigman, Phys. Rev. Lett. 42, 1117 (1979); S. Wolfram, Phys. Lett. 82B, 65 (1979); G. D. Starkman et al., Phys. Rev. D 41, 3594 (1990); D. Javorsek et al., Phys. Rev. Lett. 87, 231804 (2001); S. Mitra, Phys. Rev. D 70, 103517 (2004); G. D. Mack, J.F. Beacom, and G. Bertone, Phys. Rev. D 76, 043523 (2007); arXiv:0705.4298.

[24] B.D. Wandelt et al., arXiv:astro-ph/0006344; P.C. McGuire and P.J. Steinhardt, arXiv:astro-ph/0105567; G. Zaharijas and G. R. Farrar, Phys. Rev. D 72, 083502 (2005); arXiv:astro-ph/0406531.

[25] R. Foadi, M. T. Frandsen, T. A. Ryttov, and F. Sannino, Phys. Rev. D 76, 055005 (2007).

[26] C. Kouvaris, Phys. Rev. D 76, 015011 (2007).

[27] N. J. Evans, S. D. H. Hsu, and M. Schwetz, arXiv:hep-th/ 9707260.

[28] K. Kainulainen, K. Tuominen, and J. Virkajarvi, Phys. Rev. D 75, 085003 (2007).

[29] D. S. Akerib et al. (CDMS Collaboration), Phys. Rev. Lett. 93, 211301 (2004).

[30] C. Kouvaris, Phys. Rev. D 77, 023006 (2008).

[31] J. A. Harvey and M. S. Turner, Phys. Rev. D 42, 3344
(1990).

[32] S. M. Barr, R. S. Chivukula, and E. Farhi, Phys. Lett. B 241, 387 (1990).

[33] S. Y. Khlebnikov and M.E. Shaposhnikov, Phys. Lett. B 387, 817 (1996).

[34] R. S. Chivukula and T. P. Walker, Nucl. Phys. B329, 445 (1990).

[35] M.L. Burns and R. V. E. Lovelace, Astrophys. J. 262, 87 (1982).

[36] F. A. Agaronian and V. V. Vardanian, Report No. eFI-82754-85-YEREVAN, 1985.

[37] M. Y. Khlopov, Cosmoparticle Physics (World Scientific, Singapore, 1999).

[38] M. Pospelov, Phys. Rev. Lett. 98, 231301 (2007).

[39] M. Kaplinghat and A. Rajaraman, Phys. Rev. D 74, 103004 (2006).

[40] K. Kohri and F. Takayama, Phys. Rev. D 76, 063507 (2007); arXiv:hep-ph/0605243.

[41] K. Hamaguchi, T. Hatsuda, M. Kamimura, Y. Kino, and T. T. Yanagida, Phys. Lett. B 650, 268 (2007).

[42] M. Kawasaki, K. Kohri, and T. Moroi, Phys. Lett. B 649, 436 (2007).

[43] K. Jedamzik, arXiv:0707.2070 [Phys. Rev. D (to be published)].

[44] J. Pradler and F. D. Steffen, arXiv:0710.2213.

[45] K. Jedamzik, arXiv:0710.5153.

[46] M. Pospelov, arXiv:0712.0647.

[47] M. Khlopov, Y. Levitan, E. Sedelnikov, and I. Sobol, Phys. At. Nucl. 57, 1393 (1994).

[48] E. V. Sedelnikov, S. S. Filippov, and M. Y. Khlopov, Phys. At. Nucl. 58, 235 (1995).

[49] M. Kawasaki, K. Kohri, and T. Moroi, Phys. Lett. B 625, 7 (2005); arXiv:astro-ph/0402490.

[50] M. Kawasaki, K. Kohri, and T. Moroi, Phys. Rev. D 71, 083502 (2005); arXiv:astro-ph/0408426.

[51] K. Kohri, T. Moroi, and A. Yotsuyanagi, Phys. Rev. D 73, 123511 (2006); arXiv:hep-ph/0507245.

[52] K. Jedamzik, Phys. Rev. D 74, 103509 (2006).

[53] P. Mueller, Phys. Rev. Lett. 92, 022501 (2004); arXiv:nucl-ex/0302025.

[54] P. F. Smith and J. R. J. Bennet, Nucl. Phys. B149, 525 (1979); P. F. Smith et al., Nucl. Phys. B206, 333 (1982); T. K. Hemmick et al., Phys. Rev. D 41, 2074 (1990); P. Verkerk et al., Phys. Rev. Lett. 68, 1116 (1992); T. Yamagata, Y. Takamori, and H. Utsunomiya, Phys. Rev. D 47, 1231 (1993).

[55] D. McCammon et al., Nucl. Instrum. Methods Phys. Res., Sect. A 370, 266 (1996); Astrophys. J. 576, 188 (2002).

[56] K. Belotsky, Yu. Bunkov, H. Godfrin, M. Khlopov, and R. Konoplich, arXiv:astro-ph/0606350.

[57] C. B. Winkelmann, Y.M. Bunkov, and H. Godfrin, Gravitation and Cosmology 11, 87 (2005).

[58] Y. Zeldovich and I. Novikov, Struktura i Evolyutsiya Vselennoi (Nauka, Moscow, 1975) [Structure and Evolution of the Universe (The University of Chicago Press, Chicago, 1983)].

[59] R. J. Scherrer and M.S. Turner, Phys. Rev. D 33, 1585 (1986).

[60] A. D. Dolgov, Phys. Rep. 370, 333 (2002). 